\title{
BERNARD LACOLLE
}

\section{Fonctions convexes et logarithmes de polynômes à coefficients positifs}

\author{
Annales de la faculté des sciences de Toulouse $\sigma^{e}$ série, tome $3, \mathrm{n}^{\circ} 1$ \\ (1994), p. 91-133 \\ <http://www.numdam.org/item?id=AFST_1994_6_3_1_91_0>
}

(C) Université Paul Sabatier, 1994, tous droits réservés.

L'accès aux archives de la revue «Annales de la faculté des sciences de Toulouse » (http://picard.ups-tlse.fr/ annales/) implique l'accord avec les conditions générales d'utilisation (http://www.numdam.org/conditions). Toute utilisation commerciale ou impression systématique est constitutive d'une infraction pénale. Toute copie ou impression de ce fichier doit contenir la présente mention de copyright.

\section{Numdam}

Article numérisé dans le cadre du programme Numérisation de documents anciens mathématiques http://www.numdam.org/ 


\title{
Fonctions convexes et logarithmes de polynômes à coefficients positifs ${ }^{(*)}$
}

\author{
BerNaRd LACOLLE ${ }^{(1)}$
}

RÉSUMÉ. - Nous présentons un procédé original permettant d'obtenir certaines fonctions convexes $f$ de plusieurs variables réelles $\left(t_{1}, \ldots, t_{d}\right)$, comme limites de suites de fonctions $\left\{f_{n}\right\}$ définies par

$$
f_{n}\left(t_{1}, \ldots, t_{d}\right)=\frac{1}{n} \log P_{n}\left(e^{t_{1}}, \ldots, e^{t_{d}}\right)
$$

où les $P_{n}$ sont des "polynômes généralisés" à coefficients positifs. Nous montrons que toute fonction convexe, propre et semi-continue inférieurement est limite ponctuelle de fonctions $f_{n}$ définies plus haut. Pour une fonction convexe d'une variable, sans autre hypothèse, la condition devient nécessaire et suffisante. Nous étudions ensuite les relations entre la polaire de $f$ et les coefficients des polynômes $P_{n}$. Dans le cas d'une variable, sur un exemple important où les polynômes $P_{n}$ sont définis comme les traces des puissances $n$-èmes de certaines matrices polynomiales, deux théorèmes de localisations des racines des polyômes $\boldsymbol{P}_{\boldsymbol{n}}$ sont établis.

Abstract. - Using an original method, we show that a large class of convex functions $f$ of several real variables $\left(t_{1}, \ldots, t_{d}\right)$, can be seen as the limits of some sequences of functions $\left\{f_{n}\right\}$ defined by

$$
f_{n}\left(t_{1}, \ldots, t_{d}\right)=\frac{1}{n} \log P_{n}\left(e^{t_{1}}, \ldots, e^{t_{d}}\right)
$$

where $P_{n}$ are "generalized polynomials" with positive coefficients. We show that any convex, proper and lower semi-continuous function is the limit of some sequences of previous functions $f_{n}$. For any convex function of one variable only, without any other hypothesis, this condition becomes necessary and sufficient. Then, we study a relation between the conjugate function of $f$ and the coefficients of the polynomials $P_{n}$. About an important example in which the polynomials are defined as the traces of the successive powers of particular matrices with polynomial coefficients, we give two theorems about the localization of the roots of the polynomials $P_{n}$.

(*) Reçu le 12 mars 1992

(1) Laboratoire LMC-IMAG, Equipe Géométrie Algorithmique, Tour IRMA, B.P. 53, F-38041 Grenoble Cedex 9 (France) 


\section{Introduction}

Dans cet article, nous étudions quelques propriétés des fonctions de $d$ variables réelles obtenues comme limites ponctuelles de suites de fonctions $\left\{f_{n}\right\}$ définies pour tout $\left(t_{1}, \ldots, t_{d}\right) \in \mathbb{R}^{d}$ par :

$$
f_{n}\left(t_{1}, \ldots, t_{d}\right)=\frac{1}{n} \log P_{n}\left(e^{t_{1}}, \ldots, e^{t_{d}}\right),
$$

où les $P_{n}$ sont des "polynômes généralisés" à coefficients positifs. L'origine de la méthode se situe en Physique Statistique dans l'étude de systèmes discrets comme le modèle d'Ising ([1], [3]). Il s'agit du cas d'une ou deux variables et les "fonctions de partitions" de la physique jouent le rôle des polynômes généralisés $P_{n}$, tandis que les fonctions $f_{n}$ et leurs limites correspondent aux fonctions "d'énergie libre" ([1]-[3], [9]). Dans un précédent article nous avons étudié, dans le cas d'une variable, la relation entre les singularités de $f$ et les zéros des polynômes $P_{n}$, dans le cas où $f$ est lipschitzienne [4]. D'autre part, en se référant aux propriétés des fonctions convexes $([5],[8])$, cette méthode donne lieu à des développements mathématiques originaux [3]. Dans le présent exposé, nous étendons certains de ces résultats au cas des fonctions convexes de plusieurs variables réelles en montrant que l'on peut obtenir toute fonction convexe propre et semicontinue inférieurement comme limite des fonctions $f_{n}$ définies plus haut. Dans le cas d'une seule variable, la propriété d'obtenir une fonction convexe quelconque comme limite ponctuelle des suites $\left\{f_{n}\right\}$ devient nécessaire et suffisante. Puis, nous portons notre attention sur les relations entre la polaire et $f$ et les coefficients des polynômes $P_{n}$ dans le cas où ces coefficients possèdent certaines propriétés de régularité.

Nous avons délibérément fait cette étude dans le cadre de la convergence ponctuelle. Nous justifions ce choix par le contexte et les motivations numériques du domaine de la Physique Statistique qui ont été à l'origine de ce travail. Les propositions et théorèmes énoncés sont vus avec les hypothèses les plus fines pour ce type de convergence, ce qui peut les rendre assez techniques. D'autre part, notre utilisation systématique du lemme 2, basé sur les rapports entre la notion de maximun et les logarithmes pondérés de sommes, nous a conduit naturellement au choix de la convergence simple. Cependant, certaines de ces notions s'interprètent, en particulier, en terme d'épi-convergence. Ceci pose un problème beaucoup plus large, qui est de savoir si on peut se passer, entre les logarithmes des polynômes et les 
fonctions convexes, de l'utilisation intermédiaire de fonctionnelles linéaires comme il est fait tout au long de cet exposé. Ce point sera évoqué dans la conclusion.

La seconde partie de l'article est de nature différente et nous y étudions le cas à une variable où les polynômes $P_{n}$ sont définis comme les traces des puissances $n$-èmes de matrices polynomiales à coefficients positifs. Un premier théorème de localisation "en secteur" des racines est donné lorsque les coefficients des matrices sont des polynômes à coefficients réels positifs. Un autre théorème de localisation est ensuite montré, pouvant s'appliquer dans un cadre plus général où les matrices ne sont pas obligatoirement à coefficients réels positifs.

1. Fonctions convexes et logarithmes de polynômes généralisés à coefficients positifs

\subsection{Définitions et notations}

Nous désignerons par $\log x$ le logarithme népérien d'un nombre réel $x$ strictement positif.

D'autre part, nous abrégerons "semi-continue inférieurement" par le sigle SCI.

Tout au long de l'exposé, $d$ désignera un entier positif, dimension de l'espace $\mathbf{R}^{d}$ des variables.

DÉfinition (Famille presque nulle) .- Pour une dimension $d$ fixée, on appelle famille presque nulle de nombres réels, une famille $A=\left\{a_{i}\right\}_{i \in \mathbf{Z}^{d}}$ de nombres réels comportant un nombre fini et non nul d'élément non nuls. Si les éléments de la famille sont positifs, on parle de famille positive presque nulle.

DÉfinitions (Polynôme généralisé, polynôme GCP) - - Le polynôme généralisé en les variables $z=\left(z_{1}, \ldots, z_{d}\right)$ associé à la famille presque nulle $A=\left\{a_{i}\right\}_{i \in \mathbf{Z}^{d}}$ est défini par

$$
\begin{gathered}
P^{A}\left(z_{1}, \ldots, z_{d}\right)=\sum_{\left(i_{1}, \ldots, i_{d}\right) \in \mathbf{Z}^{d}} z_{1}^{i_{1}} \ldots z_{d}^{i_{d}} a_{\left(i_{1}, \ldots, i_{d}\right)} . \\
-93-
\end{gathered}
$$


Si $A=\left\{a_{i}\right\}_{i \in \mathbf{Z}^{d}}$ est une famille positive presque nulle, le polynôme généralisé ainsi défini est appelé polynôme $\mathrm{GCP}$, pour désigner un polynôme Généralisé à Coefficients (réels) Positifs.

DÉfinition (Propriété $\mathcal{P}$ et procédé d'approximation $\mathcal{L P}$ ) .- Une fonction $f$ de $\mathbf{R}^{d}$ dans $\mathbf{R} \cup\{-\infty,+\infty\}$ vérifie la propriété $\mathcal{P}$ s'il existe une suite $\left\{P_{n}\right\}_{n \in \mathbb{N}}$ de polynômes $\mathrm{GCP}$, telle que pour tout $\left(t_{1}, \ldots, t_{d}\right) \in \mathbb{R}^{d}$ :

$$
f\left(t_{1}, \ldots, t_{d}\right)=\operatorname{Lim}_{n \rightarrow+\infty} \frac{1}{n} \log P_{n}\left(e^{t_{1}}, \ldots, e^{t_{d}}\right) .
$$

Dans la suite, ce procédé d'approximation est appelé approximation $\mathcal{L} \mathcal{P}$ (approximation par les Logarithmes de Polynômes).

DÉfinition (Suite $\mathcal{L P}$-convergente de familles presque nulles) .- Si, de plus, la suite précédente de polynômes GCP est associée à la suite de familles positives presque nulles $\mathcal{A}=\left\{A^{(n)}\right\}_{n \in \mathbb{N}}$ avec pour tout entier $n$, $A^{(n)}=\left\{a_{i}^{(n)}\right\}_{i \in \mathbf{Z}^{d}}$, on note

$$
P^{A^{(n)}}\left(z_{1}, \ldots, z_{d}\right)=\sum_{\left(i_{1}, \ldots, i_{d}\right) \in \mathbf{Z}^{d}} z_{1}^{i_{1}} \ldots z_{d}^{i_{d}} a_{\left(i_{1}, \ldots, i_{d}\right)}^{(n)} .
$$

$S^{\prime}$ il existe une application, que l'on notera $f^{\mathcal{A}}$, de $\mathbb{R}^{d}$ dans $\mathbf{R} \cup\{-\infty,+\infty\}$ vérifiant pour tout $\left(t_{1}, \ldots, t_{d}\right) \in \mathbb{R}^{d}$ :

$$
f^{\mathcal{A}}\left(t_{1}, \ldots, t_{d}\right)=\operatorname{Lim}_{n \rightarrow \infty} \frac{1}{n} \log P^{A^{(n)}}\left(e^{t_{1}}, \ldots, e^{t_{d}}\right),
$$

on dit que la suite $\mathcal{A}=\left\{A^{(n)}\right\}_{n \in \mathbb{N}}$ est $\mathcal{L P}$-convergente.

\subsection{Quelques propriétés préliminaires}

La propriété 1 établit la convexité des fonctions définies à l'aide de logarithmes de polynômes précédents.

PropriétÉ 1.- Pour tout polynôme GCP en les variables $z=\left(z_{1}, \ldots, z_{d}\right)$ asociée à une famille positive presque nulle $A=\left\{a_{i}\right\}_{i \in \mathbf{Z}^{d}}$ :

$$
\begin{gathered}
P^{A}\left(z_{1}, \ldots, z_{d}\right)=\sum_{\left(i_{1}, \ldots, i_{d}\right) \in \mathbf{Z}^{d}} z_{1}^{i_{1}} \ldots z_{d}^{i_{d}} a_{\left(i_{1}, \ldots, i_{d}\right)}, \\
-94-
\end{gathered}
$$


Fonctions convexes et logarithmes de polynômes à coefficients positifs

la fonction de $\mathbb{R}^{d}$ dans $\mathbb{R}$ définie par

$$
\left(t_{1}, \ldots, t_{d}\right) \rightarrow \frac{1}{n} \log P^{A}\left(e^{t_{1}}, \ldots, e^{t_{d}}\right)
$$

est une fonction convexe de $\mathbf{R}^{d}$ dans $\mathbf{R}$.

Démonstration. - Cette démonstration ne présente pas de difficulté. On la trouvera évoquée dans [8, page 325].

Pour établir le théorème 3 , nous utiliserons la propriété fondamentale que toute fonction $f$ convexe propre $S C I$ de $\mathbf{R}^{d}$ dans $\mathbb{R} \cup\{+\infty\}$ s'écrit, pour tout $t \in \mathbb{R}^{d}$,

$$
f(t)=\operatorname{Sup}_{i \in I} \ell_{i}(t)
$$

les fonctionnelles $\ell_{i}(i \in I)$ étant les fonctionnelles affines (continues) minorantes de $f$.

Afin d'obtenir le résultat du théorème 4 , nous utiliserons le cas où la fonction $f$ peut s'écrire, pour tout $t \in \mathbb{R}^{d}$,

$$
f(t)=\operatorname{Lim}_{n \rightarrow+\infty} \operatorname{Sup}_{i \in I_{n}} \ell_{i}(t),
$$

les ensembles $I_{n}$ étant des ensembles finis, mais les fonctionnelles $\ell_{i}\left(i \in I_{n}\right)$ n'étant pas forcément minorantes de $f$.

Lemme 2. - Pour tout polynôme GCP en les variables $\left(z_{1}, \ldots, z_{d}\right)$ associé à une famille positive presque nulle $A=\left\{a_{i}\right\}_{i \in \mathbf{Z}^{d}}$ :

$$
P^{A}\left(z_{1}, \ldots, z_{d}\right)=\sum_{\left(i_{1}, \ldots, i_{d}\right) \in \mathbf{Z}^{d}} z_{1}^{i_{1}} \ldots z_{d}^{i_{d}} a_{\left(i_{1}, \ldots, i_{d}\right)},
$$

on note $F$ le sous-ensemble fini de $\mathbb{Z}^{d}$ défini par

$$
F=\left\{\left(i_{1}, \ldots, i_{d}\right) \in \mathbb{Z}^{d}: a_{\left(i_{1}, \ldots, i_{d}\right)} \neq \mathbf{0}\right\},
$$

et pour tout $i=\left(i_{1}, \ldots, i_{d}\right) \in F$, on note $\ell_{i}$ la fonctionnelle affine

$$
\left(t_{1}, \ldots, t_{d}\right) \rightarrow \ell_{i}\left(t_{1}, \ldots, t_{d}\right)=\left(\frac{i_{1}}{n}\right) t_{1}+\cdots+\left(\frac{i_{d}}{n}\right) t_{d}+\frac{\log a_{\left(i_{1}, \ldots, i_{d}\right)}}{n}
$$




\section{Bernard Lacolle}

Pour tout $t=\left(t_{1}, \ldots, t_{d}\right) \in \mathbb{R}^{d}$, on $a$

$$
\begin{aligned}
\operatorname{Max}_{i \in F} \ell_{i}\left(t_{1}, \ldots, t_{d}\right) & \leq \frac{1}{n} \log P^{A}\left(e^{t_{1}}, \ldots, e^{t_{d}}\right) \leq \\
& \leq \operatorname{Max}_{i \in F} \ell_{i}\left(t_{1}, \ldots, t_{d}\right)+\frac{\log \operatorname{Card}(F)}{n} .
\end{aligned}
$$

Démonstration. - On a

$$
\begin{aligned}
& \underset{\left(i_{1}, \ldots, i_{d}\right) \in F}{\operatorname{Max}}\left\{\left(e^{t_{1}}\right)^{i_{1}} \ldots\left(e^{t_{d}}\right)^{i_{d}} a_{\left(i_{1}, \ldots, i_{d}\right)}\right\} \leq \\
& \leq \sum_{\left(i_{1}, \ldots, i_{d}\right) \in F}\left(e^{t_{1}}\right)^{i_{1}} \ldots\left(e^{t_{d}}\right)^{i_{d}} a_{\left(i_{1}, \ldots, i_{d}\right)} \leq \\
& \leq \operatorname{Card}(F) \cdot \operatorname{Max}_{\left(i_{1}, \ldots, i_{d}\right) \in F}\left\{\left(e^{t_{1}}\right)^{i_{1}} \ldots\left(e^{t_{d}}\right)^{i_{d}} a_{\left(i_{1}, \ldots, i_{d}\right)}\right\},
\end{aligned}
$$

d'où le résultat en prenant les logarithmes et en divisant par $n$. $\square$

\subsection{Les théorèmes}

THÉORÈme 3.- Toute fonction $f$ convexe propre SCI de $\mathbf{R}^{d}$ dans $\mathbf{R} \cup\{+\infty\}$ vérifie la propriété $\mathcal{P}$.

Démonstration. - Toute fonction $f$ convexe, propre SCI, de $\mathbf{R}^{d}$ dans $\mathbf{R} \cup\{+\infty\}$ s'écrit :

$$
f(t)=\operatorname{Sup}_{i \in I} \ell_{i}(t)
$$

où $\left\{\ell_{i}\right\}_{i \in I}$ constitue l'ensemble des fonctionnelles affines minorantes de $f$ que nous écrirons ici sous la forme :

$$
\ell_{i}\left(t_{1}, \ldots, t_{d}\right)=u_{i}^{1} t_{1}+\cdots+u_{i}^{d} t_{d}-u_{i}^{d+1}, \quad\left(t_{1}, \ldots, t_{d}\right) \in \mathbb{R}^{d}
$$

avec

$$
u_{i}=\left(u_{i}^{1}, \ldots, u_{i}^{d}, u_{i}^{d+1}\right) \in \mathbb{R}^{d+1}, \quad i \in I
$$

Pour tout $n \in \mathbb{N}$, on désigne par $I_{n}$ les sous-ensembles de $I$ tels que les fonctionnelles affines $\ell_{i}\left(i \in I_{n}\right)$ vérifient

$$
\left|u_{i}^{k}\right| \leq n, \quad 1 \leq k \leq d+1 .
$$


Il est facile de montrer qu'en définissant $f_{n}$, pour tout $t \in \mathbb{R}^{d}$, par

$$
f_{n}(t)=\operatorname{Sup}_{i \in I_{n}} \ell_{i}(t)
$$

alors pour tout $t \in \mathbb{R}^{d}$ :

$$
f(t)=\operatorname{Lim}_{n \rightarrow+\infty} f_{n}(t)
$$

Considérons l'ensemble $J^{(n)}$ des $(d+1)$-uplets $\left(i_{1}, \ldots, i_{d+1}\right)$ de $\mathbb{Z}^{d+1}$ tels que :

i) $-n^{2} \leq i_{k} \leq n^{2}(1 \leq k \leq d+1)$;

i) la fonctionnelle affine

$$
\left(t_{1}, \ldots, t_{d}\right) \rightarrow\left(\frac{i_{1}}{n}\right) t_{1}+\cdots+\left(\frac{i_{d}}{n}\right) t_{d}-\frac{i_{d+1}}{n}
$$

est telle qu'il existe une fonctionnelle affine $\ell_{i}\left(i \in I_{n}\right)$ :

$$
\left(t_{1}, \ldots, t_{d}\right) \rightarrow u_{i}^{1} t_{1}+\cdots+u_{i}^{d} t_{d}-u_{i}^{d+1},
$$

vérifiant

$$
\left|\frac{i_{k}}{n}-u_{i}^{k}\right| \leq \frac{1}{n}, \quad 1 \leq k \leq d+1
$$

iii) s'il existe plusieurs éléments $\left(i_{1}, \ldots, i_{d+1}\right)$ vérifiant i) et ii) et possédant les mêmes $d$ premiers indices $\left(i_{1}, \ldots, i_{d}\right)$, on ne retient que celui dont le $(d+1)$-ème indice et minimum.

On notera $\lambda_{\left(i_{1}, \ldots, i_{d}\right)}$ la fonctionnelle affine :

$$
\lambda_{\left(i_{1}, \ldots, i_{d}\right)}\left(t_{1}, \ldots, t_{d}\right)=\left(\frac{i_{1}}{n}\right) t_{1}+\cdots+\left(\frac{i_{d}}{n}\right) t_{d}-\frac{i_{d+1}}{n} .
$$

En notant $F^{(n)}$ le sous-ensemble des $\left(i_{1}, \ldots, i_{d}\right)$ de $\mathbb{Z}^{d}$ tel qu'il existe $\left(i_{1}, \ldots, i_{d+1}\right)$ appartenant à $J^{(n)}$, un calcul direct montre que pour tout $t \in \mathbb{R}^{d}$ :

$$
\left|\operatorname{Sup}_{i \in I_{n}} \ell_{i}(t)-\operatorname{Max}_{i \in F^{(n)}} \lambda_{i}(t)\right| \leq \frac{\left(1+\|t\|_{1}\right)}{n} .
$$

On en déduit, en posant

$$
\begin{aligned}
g_{n}(t) & =\underset{i \in F^{(n)}}{\operatorname{Max}} \lambda_{i}(t) \\
& -97-
\end{aligned}
$$


que pour tout $t \in \mathbb{R}^{d}$

$$
f(t)=\operatorname{Lim}_{n \rightarrow+\infty} g_{n}(t)
$$

Considérons pour tout $n \in \mathbb{N}$ le polynôme $P_{n}$ :

$$
P_{n}\left(z_{1}, \ldots, z_{d}\right)=\sum_{\left(i_{1}, \ldots, i_{d}\right) \in F^{(n)}} z_{1}^{i_{1}} \ldots z_{d}^{i_{d}} e^{-i_{d+1}} .
$$

D'après le lemme 2, la définition des fonctionnelles $\lambda_{i}$ et des fonctions $g_{n}$, il vient

$$
\begin{aligned}
g_{n}\left(t_{1}, \ldots, t_{d}\right) & \leq \frac{1}{n} \log P_{n}\left(e^{t_{1}}, \ldots, e^{t_{d}}\right) \leq \\
& \leq g_{n}\left(t_{1}, \ldots, t_{d}\right)+\frac{\log \operatorname{Card}\left(F^{(n)}\right)}{n} .
\end{aligned}
$$

D'autre part, nous avons

$$
\operatorname{Card}\left(F^{(n)}\right) \leq\left(2 n^{2}+1\right)^{d}
$$

ce qui permet de conclure que pour tout $\left(t_{1}, \ldots, t_{d}\right) \in \mathbb{R}^{d}$ :

$$
f\left(t_{1}, \ldots, t_{d}\right)=\operatorname{Lim}_{n \rightarrow+\infty} \frac{1}{n} \log P_{n}\left(e^{t_{1}}, \ldots, e^{t_{d}}\right),
$$

d'où le résultat.

Il est à noter que d'après la propriété 1 , les fonctions

$$
\left(t_{1}, \ldots, t_{d}\right) \rightarrow \frac{1}{n} \log P_{n}\left(e^{t_{1}}, \ldots, e^{t_{d}}\right)
$$

sont convexes.

ThÉoRÈme 4. - Une fonction de $\mathbb{R}$ dans $\mathbb{R} \cup\{-\infty,+\infty\}$ vérifie la propriété $\mathcal{P}$ si et seulement si $f$ est une fonction convexe.

Note. - Une version de ce théorème a d'abord été établie pour les fonctions convexes lipschitziennes de $R$ dans $\mathbb{R}$ et publiée dans [4]. Avec les mêmes outils de démonstration, nous étendons le théorème à des fonctions convexes quelconques de $\mathbb{R}$ dans $\mathbb{R} \cup\{-\infty,+\infty\}[\mathbf{3}]$. I faut noter le caractère constructif de cette méthode de démonstration qui nous permet de traiter géométriquement le cas d'une fonction convexe quelconque. 
Démonstration. - D'après la propriété 1 , nous savons déjà que si $P_{n}$ est un polynôme GCP, toute fonction définie par

$$
t \rightarrow \frac{1}{n} \log P_{n}\left(e^{t}\right)
$$

est convexe. Par passage à la limite, on en déduit la convexité de $f$ vérifiant la propriété $\mathcal{P}$.

Nous commençons à établir la réciproque dans le cas d'une fonction convexe de $\mathbf{R}$ dans $\mathbb{R}$, à l'aide d'une méthode constructive qui permettra de traiter le cas général.

Considérons donc une fonction convexe de $R$ dans $R: f$ est donc finie et continue sur $\mathbb{R}$. Soit $t$ un nombre réel fixé et $\varepsilon$ un nombre réel strictement positif.

Pour tout $n \in \mathbb{N}$, on désigne par $I_{n}$ l'intervalle $[-\log n, \log n]$ et on divise $I_{n}$ en $n$ sous-intervalles égaux. On pose

$$
t_{i}^{n}=-\log n+2 i \frac{\log n}{n}, \quad i=0,1, \ldots, n .
$$

Choisissons $N_{1}$ tel que $t$ appartienne à l'intérieur de $I_{n}$ pour tout $n \geq N_{1}$. L'indice $s$ est défini par

$$
t \in\left[t_{s}^{n}, t_{s+1}^{n}[\right.
$$

L'indice $s$ dépend de $n$, mais nous omettons cette précision pour simplifier les notations. Pour tout $i \in\{0,1,2, \ldots, n-1\}$, on appelle $\ell_{i}^{n}$ la fonctionnelle affine qui interpole $f$ aux points $\left(t_{i}^{n}, f\left(t_{i}^{n}\right)\right)$ et $\left(t_{i+1}^{n}, f\left(t_{i+1}^{n}\right)\right)$ :

$$
\ell_{i}^{n}(u)=\frac{t_{i+1}^{n} f\left(t_{i}^{n}\right)-t_{i}^{n} f\left(t_{i+1}^{n}\right)}{t_{i+1}^{n}-t_{i}^{n}}+\frac{f\left(t_{i+1}^{n}\right)-f\left(t_{i}^{n}\right)}{t_{i+1}^{n}-t_{i}^{n}} u .
$$

Utilisant la continuité uniforme de $f$ sur tout compact de $\mathbb{R}$, nous pouvons choisir $N_{2} \geq N_{1}$ tel que pour tout $n \geq N_{2}$

$$
u, v \in\left[t_{s}^{n}, t_{s+1}^{n}\right] \Rightarrow|f(u)-f(v)| \leq \frac{\varepsilon}{3} .
$$

Considérant $t$ et utilisant la convexité de $f$, nous pouvons donc écrire

$$
0 \leq \ell_{s}^{n}(t)-f(t) \leq \operatorname{Max}\left\{f\left(t_{s}^{n}\right), f\left(t_{s+1}^{n}\right)\right\}-f(t) \leq \frac{\varepsilon}{3},
$$

et pour tout $i \neq s$

$$
\begin{gathered}
\ell_{i}^{n}(t)-f(t) \leq 0 \\
-99-
\end{gathered}
$$




\section{Bernard Lacolle}

d'où :

$$
0 \leq\left(\underset{i=0,1, \ldots, n-1}{\operatorname{Max}} \ell_{i}^{n}(t)\right)-f(t) \leq \frac{\varepsilon}{3} .
$$

Remplaçons maintenant les $\ell_{n}^{i}(t)$ par les approximations suivantes :

$$
\lambda_{i}^{n}(t)=\frac{t_{i+1}^{n} f\left(t_{i}^{n}\right)-t_{i}^{n} f\left(t_{i+1}^{n}\right)}{t_{i+1}^{n}-t_{i}^{n}}+\frac{p_{i}^{n}}{n} t
$$

avec

$$
p_{i}^{n}=\left\lfloor n \frac{f\left(t_{i+1}^{n}\right)-f\left(t_{i}^{n}\right)}{t_{i+1}^{n}-t_{i}^{n}}\right\rfloor
$$

( $\lfloor x\rfloor$ désigne la partie entière de $x$ ).

Il est facile de montrer que pour tout $i \in\{0,1, \ldots, n-1\}$ :

$$
\left|\ell_{i}^{n}(t)-\lambda_{i}^{n}(t)\right| \leq \frac{|t|}{n} \leq \frac{\log n}{n} .
$$

Choisissons maintenant un entier $N_{3} \geq N_{2}$ tel que pour tout $n \geq N_{3}$ on ait

$$
\frac{\log n}{n} \leq \frac{\varepsilon}{3} \text {. }
$$

Si $n \geq N_{3}$, nous avons donc l'inégalité suivante pour tout $i \in\{0,1, \ldots, n-1\}$

$$
-\frac{\varepsilon}{3}+\lambda_{i}^{n}(t) \leq \ell_{i}^{n}(t) \leq \lambda_{i}^{n}(t)+\frac{\varepsilon}{3},
$$

et donc :

$$
\left|\underset{i=0,1, \ldots, n-1}{\operatorname{Max}} \ell_{i}^{n}(t)-\underset{i=0,1, \ldots, n-1}{\operatorname{Max}} \lambda_{i}^{n}(t)\right| \leq \frac{\varepsilon}{3} .
$$

Écrivons $\lambda_{i}^{n}(t)$ sous la forme $\lambda_{i}^{n}(t)=\alpha_{i}^{(n)}+t\left(p_{i}^{n} / n\right)$, et considérons la suite de polynômes GCP :

$$
P_{n}(z)=\sum_{i=0}^{n-1} z^{p_{i}^{n}} e^{n \alpha_{i}^{(n)}},
$$

vérifiant

$$
\begin{aligned}
P_{n}\left(e^{t}\right) & =\sum_{i=0}^{n-1} e^{n \lambda_{i}^{n}(t)} . \\
& -100-
\end{aligned}
$$


Nous avons d'après le lemme 2 :

$$
\underset{i=0,1, \ldots, n-1}{\operatorname{Max}} \lambda_{i}^{n}(t) \leq \frac{1}{n} \log \sum_{i=0}^{n-1} e^{n \lambda_{i}^{n}(t)} \leq \underset{i=0,1, \ldots, n-1}{\operatorname{Max}} \lambda_{i}^{n}(t)+\frac{\log n}{n},
$$

et nous avons donc pour $n \geq N_{3}$ :

$$
\underset{i=0,1, \ldots, n-1}{\operatorname{Max}} \lambda_{i}^{n}(t) \leq \frac{1}{n} \log P_{n}\left(e^{t}\right) \leq \underset{i=0,1, \ldots, n-1}{\operatorname{Max}} \lambda_{i}^{n}(t)+\frac{\varepsilon}{3} .
$$

Des inégalité (2), (4) et (5), nous en déduisons que pour tout $n \geq N_{3}$ :

$$
\left|f(t)-\frac{1}{n} \log P_{n}\left(e^{t}\right)\right| \leq \varepsilon
$$

et donc que la suite de fonctions $\left\{(1 / n) \log P_{n}\right\}$ converge ponctuellement vers $f$, ce qui termine la démonstration pour une fonction convexe de $\mathbb{R}$ dans $\mathbb{R}$.

Traitons maintenant la réciproque dans le cas d'une fonction convexe de IR dans $\mathbf{R} \cup\{-\infty,+\infty\}$. Commençons par considérer une fonction $f$ dont le domaine effectif est un intervalle d'intérieur non vide de l'une des formes suivantes :

$$
[a, b], \quad[a, b[, \quad] a, b], \quad] a, b[, \quad[a,+\infty[\text { ou }] a,+\infty[,
$$

que nous noterons naturellement $[a, \beta)$ ou $] a, \beta)$.

Nous allons examiner, uniquement au point $a$, comment modifier la méthode précédente pour obtenir un résultat général. Avec des notations équivalentes, nous posons

$$
t_{i}^{n}=a+i h_{n}, \quad i=0,1, \ldots, n,
$$

le pas $h_{n}$ étant égal par exemple à $(b-a) / n$ si l'intervalle est borné, et $\log n / n$ si l'intervalle n'est pas borné.

Pour $i=1, \ldots, n-2$, les fonctionnelles affines $\ell_{i}^{n}$ sont définies de la même manière afin d'interpoler $f$ aux points $\left(t_{i}^{n}, f\left(t_{i}^{n}\right)\right)$ et $\left(t_{i+1}^{n}, f\left(t_{i+1}^{n}\right)\right)$. Nous allons redéfinir la fonctionnelle affine $\ell_{0}^{n}$ et éventuellement créer une nouvelle fonctionnelle affine $\ell_{-1}^{n}$. Pour un traitement analogue au point $b$, il nous faudrait de même redéfinir la fonctionnelle $\ell_{n-1}^{n}$ et éventuellement créer une nouvelle fonctionelle $\ell_{n}^{n}$. 
Cas $I=[a, \beta)($ figure 1$)$

Que $f$ soit continue ou non à droite de $a$, nous définissons toujours $\ell_{0}^{n}$ de façon à interpoler $f$ aux points $m_{0}^{n}=\left(t_{0}^{n}, f\left(t_{0}^{n}\right)\right)$ et $m_{1}^{n}=\left(t_{1}^{n}, f\left(t_{1}^{n}\right)\right)$. D'autre part, pour assurer la convergence vers $+\infty$ à gauche de $a$, nous créons une nouvelle fonctionnelle $\ell_{-1}^{n}$ remplissant les conditions suivantes :

- $\ell_{-1}^{n}$ est de pente négative, inférieure à celle de $\ell_{0}^{n}$ et tendant vers $-\infty$ avec $n$;

- $\ell_{-1}^{n}\left(t_{0}^{n}\right)$ est égal à $\ell_{0}^{n}\left(t_{0}^{n}\right)=\ell_{0}^{n}(a)=f(a)$.

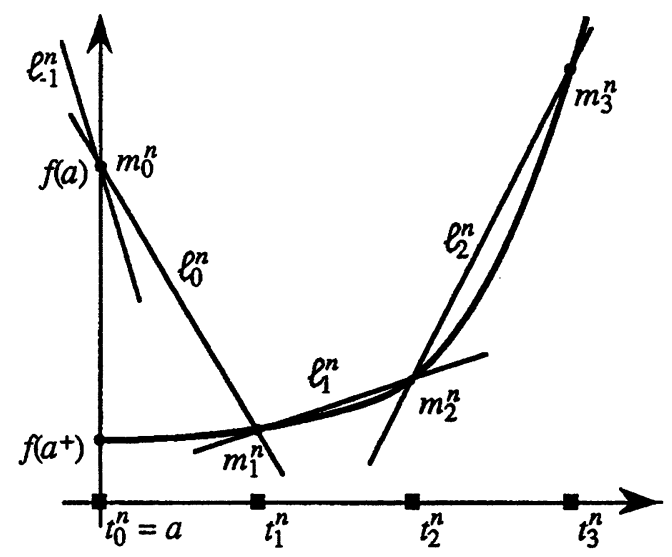

Figure 1

Cas $I=] a, \beta)$ (figures 2)

i) Si $\operatorname{Lim}_{t \rightarrow a^{+}} f(t)$ est finie (fig. 2.1), on redéfinit $\ell_{0}^{n}$ afin de vérifier les conditions suivantes :

- $\ell_{0}^{n}$ est de pente négative, inférieure à celle de $\ell_{1}^{n}$ et tendant vers $-\infty$ avec $n$,

- $\ell_{0}^{n}\left(t_{0}^{n}\right)=\ell_{0}^{n}(a)$ prend une valeur qui tend vers l'infini avec $n$.

ii) $\mathrm{Si} \operatorname{Lim}_{t \rightarrow a^{+}} f(t)=+\infty$ (fig. 2.2), nous ne prenons pas en compte la fonctionnelle $\ell_{0}^{n}$ et il suffit de considérer comme "première" fonctionnelle, la fonctionnelle $\ell_{1}^{n}$ interpolant $f$ aux points $m_{1}^{n}=\left(t_{1}^{n}, f\left(t_{1}^{n}\right)\right)$ et $m_{2}^{n}=\left(t_{2}^{n}, f\left(t_{2}^{n}\right)\right)$. 
Fonctions convexes et logarithmes de polynômes à coefficients positifs

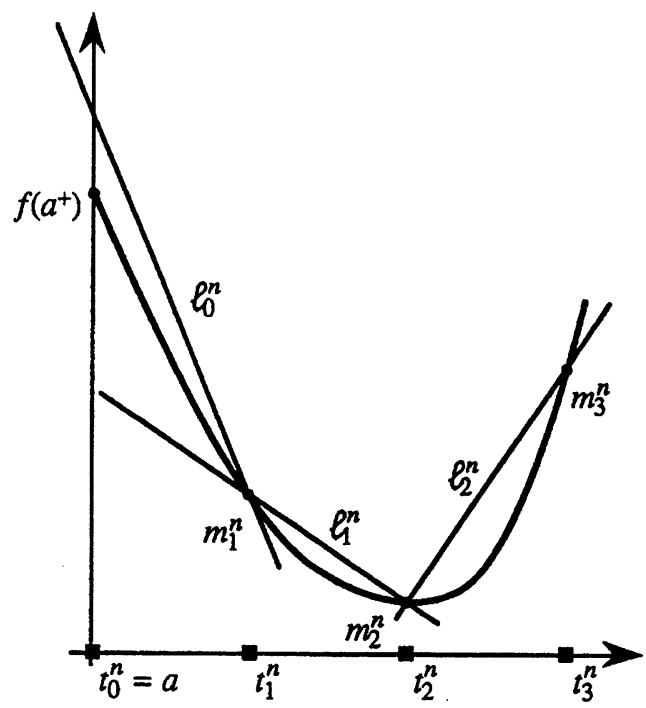

Figure 2.1

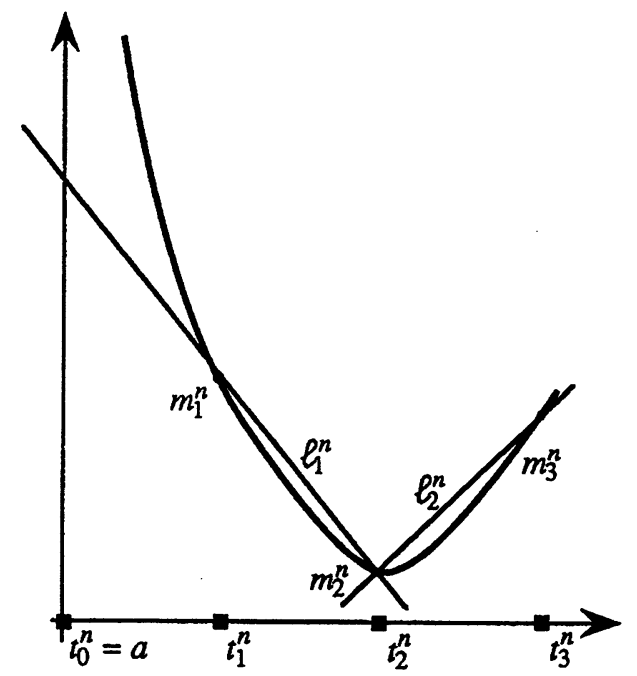

Figure 2.2

On complète de façon symétrique, l'ensemble des fonctionnelles affines en examinant le cas du point $b$. Les fonctionnelles $\lambda_{i}^{n}$ sont définies pour $i \in I^{(n)}$, $I^{(n)}$ étant un ensemble d'indices inclus dans $\{-1,0,1,2, \ldots, n-1, n\}$, par des formules analogues aux formules (3) et écrites sous la forme

$$
\lambda_{i}^{n}(t)=\alpha_{i}^{(n)}+t \frac{p_{i}^{n}}{n}, \quad i \in I^{(n)} .
$$


D'après la construction précédente, il est aisé de montrer que pour tout $t \in \mathbb{R}$ :

$$
f(t)=\operatorname{Lim}_{n \rightarrow+\infty} \operatorname{Max}_{i \in I^{(n)}} \lambda_{i}^{n}(t) .
$$

On considère également la suite de polynôme GCP :

$$
P_{n}(z)=\sum_{i \in I^{(n)}} z^{p_{i}^{n}} e^{n \alpha_{i}^{(n)}}
$$

et par utilisation du lemme 2, on montre sans difficulté que la suite de fonctions $\left\{(1 / n) \log P_{n}\right\}$ converge ponctuellement vers $f$.

On traite de façon similaire le cas où le domaine de $f$ est un intervalle d'intérieur non vide de l'une des formes suivantes :

$$
]-\infty, b[, \quad]-\infty, b]
$$

Dans le cas où $\operatorname{dom}(f)$ est réduit à un point $\{a\}$, on vérifie aisément qu'il suffit de prendre par exemple :

$$
P_{n}(z)=z^{n^{2}} e^{n f(a)-n^{2} a}+z^{-n^{2}} e^{n f(a)+n^{2} a} .
$$

Si la fonction $f$ est identiquement égale à $+\infty$, on prend par exemple :

$$
P_{n}(z)=e^{n^{2}}
$$

et si $f$ est identiquement égale à $-\infty$, on peut choisir

$$
P_{n}(z)=e^{-n^{2}}
$$

L'énumération des cas termine la démonstration.

\subsection{Quelques remarques sur le procédé d'approximation $\mathcal{L P}$}

\subsubsection{Convergence uniforme sur tout compact}

En appliquant une propriété générale des fonctions convexes [8], on en déduit aisément que la convergence est uniforme sur tout compact inclus dans l'intérieur de $\operatorname{dom}(f)$. 


\subsubsection{Procédé d'approximation $\mathcal{L P}$ et l'épi-convergence}

Le déroulement de ce travail, nous a conduit à établir nos résultats dans le cas de la convergence ponctuelle. Cette étude a commencé par le cas d'une variable et une caractérisation complète des fonctions convexes quelconques a été le premier résultat obtenu. Ce résultat ne peut être acquis par utilisation de l'épi-convergence telle qu'elle est considérée dans [10], par exemple, puisque la fonction limite du théorème 4 n'est pas SCI. D'autre part, l'utilisation de la convergence ponctuelle nous a été fortement suggérée par le lemme 2. Cependant, sous certaines hypothèses, l'utilisation de l'épi-convergence permettrait d'établir des résultats peutêtre plus simples techniquement. Ceci est le cas du théorème 3, qui établit un résultat concernant les fonctions convexes, propres et SCI dans le cas de plusieurs variables, la convergence des épigraphes étant dans ce cas vérifiée. On pourrait retrouver d'ailleurs certaines équivalences entre les deux types de convergences en faisant des hypothèses restrictives adéquates ([7], [10]). Cependant, il semble que la question importante est de savoir si on peut se passer des fonctionnelles affines intermédiaires, et s'il est donc possible de travailler directement sur les polynômes et leurs coefficients. Nous reviendrons brièvement sur ce point en conclusion, à la lumière du théorème 10 qui donne quelques éléments concernant les relations entre les coefficients des polynômes et les limites obtenues.

1.4.3. Vers un théorème de caractérisation dans le cas général de plusieurs variables

Dans le cas de plusieurs variables, une méthode générale constructive analogue à celle du théorème 4 , pourrait dans le principe, être développée même si cela pose des difficultés techniques. Cependant, on remarque, que c'est probablement une étude fine de la géométrie de la frontière du domaine effectif des fonctions et du comportement des fonctions à la frontière de ce domaine, que résident les éléments éventuels d'une caractérisation du sousensemble des fonctions convexes vérifiant la propriété $\mathcal{P}$.

\subsubsection{Approximation des singularités d'une fonction convexe lipschit-} zienne d'une variable par le procédé d'approximation $\mathcal{L P}$

Dans le procédé d'approximation $\mathcal{L P}$ d'une fonction convexe d'une variable, une relation intuitive semble relier les singularités de $f$ et la "proximité" des zéros complexes des polynômes $P_{n}$. Cette notion a été précisée dans un précédent article [4]. Nous la rappelons brièvement. 
On dit que $t_{c}$ est une singularité de la fonction $f$ de $R$ dans $R$ si la fonction de la variable réelle $f$ n'est pas analytique au voisinage de $t_{c}$.

ThÉORÈME 5 (rappel [4]). - Soit $f$ une fonction convexe lipschitzienne de $\mathbf{R}$ dans $\mathbb{R}$ et $\left\{P_{n}\right\}_{n \in \mathbb{N}}$ une suite de polynômes GCP telle que pour tout $t \in \mathbb{R}$ :

$$
f(t)=\operatorname{Lim}_{n \rightarrow \infty} \frac{1}{n} \log P_{n}\left(e^{t}\right) .
$$

Soit $\mathcal{R}$ l'ensemble de racines des polynômes $\left\{P_{n}\right\}_{n \in \mathbb{N}}$, c'est-à-dire

$$
\mathcal{R}=\bigcup_{n \in \mathbb{N}}\left\{z \in \mathbb{C}: P_{n}(z)=\mathbf{0}\right\}
$$

Si $t_{c}$ est une singularité de $f$, alors $z_{c}=e^{t_{c}}$ est un point d'accumulation de $\mathcal{R}$.

La démonstration de ce théorème est longue et a été développée dans [4].

Exemple. - On illustre le théorème précédent par l'exemple simple des polynômes généralisés :

$$
P_{n}(z)=z^{n}+z^{-n}
$$

II est facile de voir que pour tout $t \in \mathbb{R}$ :

$$
|t|=\operatorname{Lim}_{n \rightarrow \infty} \frac{1}{n} \log P_{n}\left(e^{t}\right) .
$$

Les racines de $P_{n}(z)$ sont uniformément réparties sur le cercle unité, $t_{c}=0$ est l'unique singularité de $f(t)=|t|$ et $z_{c}=e^{t_{c}}=1$ est l'unique intersection du cercle unité avec l'axe réel positif.

1.4.5. Un procédé d'approximation par des fonctions analytiques respectant la convexité

On remarque que les fonctions

$$
\frac{1}{n} \log P_{n}\left(e^{t_{1}}, \ldots, e^{t_{d}}\right),
$$

qui interviennent dans l'approximation $\mathcal{L P}$, sont des fonctions convexes analytiques. Pour une fonction convexe $f$ de $\mathbb{R}$ dans $\mathbb{R}$ donnée sur un ensemble de points

$$
\begin{gathered}
\left(t_{i}, f\left(t_{i}\right)\right), \quad i=1,2, \ldots, n, \\
-106-
\end{gathered}
$$


de la même façon que nous avons construit la suite de polynômes GCP dans le théorème 4 , nous pouvons construire des polynômes $P_{n}$ et les fonctions correspondantes $(1 / n) \log P_{n}\left(e^{t}\right)$ sont des approximations analytiques convexes de $f$. Dans le cas de plusieurs variables, une méthode constructive pourrait être envisagée.

I.5. Opération sur les suite $\mathcal{L P}$-convergentes de familles positives presque nulles

DÉFINITION .- Utilisant les notations du paragraphe 1.1, étant donné deux suites de familles positives presque nulles

$$
\mathcal{A}=\left\{A^{(n)}\right\}_{n \in \mathbb{N}} \quad \text { et } \quad \mathcal{B}=\left\{B^{(n)}\right\}_{n \in \mathbb{N}},
$$

on définit de façon naturelle les opérations suivantes :

$$
\begin{aligned}
& \mathcal{A}+\mathcal{B}=\mathcal{C}=\left\{C^{(n)}\right\}_{n \in \mathbb{N}}: c_{i}^{(n)}=a_{i}^{(n)}+b_{i}^{(n)}, n \in \mathbb{N}, i \in \mathbb{Z}^{d} ; \\
& \operatorname{Max}\{\mathcal{A}, \mathcal{B}\}=\mathcal{C}=\left\{C^{(n)}\right\}_{n \in \mathbb{N}}: c_{i}^{(n)}=\operatorname{Max}\left\{a_{i}^{(n)}, b_{i}^{(n)}\right\}, n \in \mathbb{N}, i \in \mathbb{Z}^{d} ; \\
& \mathcal{A} \otimes \mathcal{B}=\mathcal{C}=\left\{C^{(n)}\right\}_{n \in \mathbb{N}}: c_{i}^{(n)}=\sum_{j+k=i} a_{j}^{(n)} b_{k}^{(n)}, n \in \mathbb{N}, i \in \mathbb{Z}^{d} ; \\
& \mathcal{A} \hat{\nabla} \mathcal{B}=\mathcal{C}=\left\{C^{(n)}\right\}_{n \in \mathbb{N}}: c_{i}^{(n)}=\operatorname{Max}_{j+k=i} a_{j}^{(n)} b_{k}^{(n)}, n \in \mathbb{N}, i \in \mathbb{Z}^{d} ; \\
& \operatorname{Min}\{\mathcal{A}, \mathcal{B}\}=\mathcal{C}=\left\{C^{(n)}\right\}_{n \in \mathbb{N}}: c_{i}^{(n)}=\operatorname{Min}\left\{a_{i}^{(n)}, b_{i}^{(n)}\right\}, n \in \mathbb{N}, i \in \mathbb{Z}^{d} .
\end{aligned}
$$

PROPRIÉTÉS 6

i) Si $\mathcal{A}$ et $\mathcal{B}$ sont deux suites $\mathcal{L P}$-convergentes de familles positives presque nulles, alors $\mathcal{A}+\mathcal{B}, \operatorname{Max}\{\mathcal{A}, \mathcal{B}\}$ et $\mathcal{A} \otimes \mathcal{B}$ sont des suites $\mathcal{L P}$-convergentes de familles positives presque nulles, et on a:

$$
f^{\mathcal{A}+\mathcal{B}}=f^{\operatorname{Max}\{\mathcal{A}, \mathcal{B}\}}=\operatorname{Max}\left\{f^{\mathcal{A}}, f^{\mathcal{B}}\right\} \quad \text { et } f^{\mathcal{A} \otimes \mathcal{B}}=f^{\mathcal{A}}+f^{\mathcal{B}} .
$$

ii) Si $\mathcal{A}, \mathcal{B}, \mathcal{A} \hat{\nabla} \mathcal{B}$ et $\operatorname{Min}\{\mathcal{A}, \mathcal{B}\}$ sont des suites $\mathcal{L} \mathcal{P}$-convergentes de familles positives presque nulles, on a

$$
f^{\mathcal{A} \widehat{\nabla} \mathcal{B}} \leq f^{\mathcal{A}}+f^{\mathcal{B}} \quad \text { et } \quad f^{\operatorname{Min}\{\mathcal{A}, \mathcal{B}\}} \leq \operatorname{Min}\left\{f^{\mathcal{A}}, f^{\mathcal{B}}\right\} .
$$

iii) Si les nombres d'éléments non nuls de $A^{(n)}$ et $B^{(n)}$ notés respectivement $\mu_{A}^{(n)}$ et $\mu_{B}^{(n)}$ vérifient

$$
\begin{aligned}
& \frac{\log \mu_{A}^{(n)}}{n} \underset{n \rightarrow \infty}{\longrightarrow} 0 \text { ou } \frac{\log \mu_{B}^{(n)}}{n} \underset{n \rightarrow \infty}{\longrightarrow} 0 \\
&-107-
\end{aligned}
$$


et si $\mathcal{A}$ et $\mathcal{B}$ sont deux suites $\mathcal{L} \mathcal{P}$-convergentes de familles positives presque nulles, alors $\mathcal{A} \widehat{\nabla} \mathcal{B}$ est une suite $\mathcal{L P}$-convergente de familles positives presque nulles et on a la propriété

$$
f^{\mathcal{A} \widehat{\nabla} \mathcal{B}}=f^{\mathcal{A}}+f^{\mathcal{B}} .
$$

Démonstration. - Pour un multi-indice $i=\left(i_{1}, \ldots, i_{d}\right)$ de $\mathbb{Z}^{d}$ et $z=\left(z_{1}, \ldots, z_{d}\right)$ appartenant à $\left(\mathbb{R}_{+}^{*}\right)^{d}$, on note de façon naturelle

$$
z^{i}=\prod_{j=1, \ldots, d} z_{j}^{i_{j}}
$$

En ce qui concerne les égalités de i), il est tout d'abord utile et facile d'établir que si $\left\{u_{n}\right\}$ et $\left\{v_{n}\right\}$ sont deux suites de nombres réels positifs telles que $\log u_{n} / n$ et $\log v_{n} / n$ convergent respectivement vers $\alpha_{u}$ et $\alpha_{v}$ alors la suite $\left\{\left(\log \operatorname{Max}\left\{u_{n}, v_{n}\right\}\right) / n\right\}$ converge vers $\operatorname{Max}\left\{\alpha_{u}, \alpha_{v}\right\}$. L'inégalité

$$
\begin{aligned}
\operatorname{Max}\left\{\frac{\log u_{n}}{n}, \frac{\log v_{n}}{n}\right\} & \leq \frac{\log \left(u_{n}+v_{n}\right)}{n} \leq \\
& \leq \frac{\log \left(2 \operatorname{Max}\left\{u_{n}, v_{n}\right\}\right)}{n},
\end{aligned}
$$

nous permet d'affirmer que la suite $\left\{\left(\log \left(u_{n}+v_{n}\right)\right) / n\right\}$ converge vers cette même limite. $L$ 'application directe de cette dernière propriété nous permet d'affirmer que $\mathcal{A}+\mathcal{B}$ est $\mathcal{L} P$-convergente et que

$$
f^{\mathcal{A}+\mathcal{B}}=\operatorname{Max}\left\{f^{\mathcal{A}}, f^{\mathcal{B}}\right\} .
$$

De plus, l'inégalité :

$$
\begin{aligned}
\operatorname{Max}\left\{\sum_{i \in \mathbf{Z}^{d}} a_{i}^{(n)} z^{i}, \sum_{i \in \mathbf{Z}^{d}} b_{i}^{(n)} z^{i}\right\} & \leq \sum_{i \in \mathbf{Z}^{d}} \operatorname{Max}\left\{a_{i}^{(n)}, b_{i}^{(n)}\right\} z^{i} \leq \\
& \leq \sum_{i \in \mathbf{Z}^{d}}\left\{a_{i}^{(n)}+b_{i}^{(n)}\right\} z^{i}
\end{aligned}
$$

nous permet de montrer que $\operatorname{Max}\{\mathcal{A}, \mathcal{B}\}$ est $\mathcal{L P}$-convergente et que

$$
f^{\operatorname{Max}\{\mathcal{A}, \mathcal{B}\}}=\operatorname{Max}\left\{f^{\mathcal{A}}, f^{\mathcal{B}}\right\}=f^{\mathcal{A}+\mathcal{B}} .
$$


Fonctions convexes et logarithmes de polynômes à coefficients positifs

L'inégalité

$$
f^{\mathcal{A} \otimes \mathcal{B}}=f^{\mathcal{A}}+f^{\mathcal{B}}
$$

résulte directement de la définition de la multiplication des polynômes.

Les inégalités de ii) ne présentent aucune difficulté. Signalons simplement, pour l'opération Min, que la condition d'existence d'un élément non nul des familles n'est pas toujours vérifiée.

Examinons brièvement la propriété iii). On a

$$
\begin{aligned}
\sum_{i \in \mathbf{Z}^{d}} \operatorname{Max}_{j+k=i} a_{j}^{(n)} b_{k}^{(n)} z^{i} & \leq \sum_{i \in \mathbf{Z}^{d}} \sum_{j+k=i} a_{j}^{(n)} b_{k}^{(n)} z^{i} \leq \\
& \leq \operatorname{Min}\left(\mu_{A}^{(n)}, \mu_{B}^{(n)}\right) \sum_{i \in \mathbf{Z}^{d}}\left(\underset{j+k=i}{\operatorname{Max}} a_{j}^{(n)} b_{k}^{(n)}\right) z^{i},
\end{aligned}
$$

et en posant $\mathcal{A} \hat{\nabla} \mathcal{B}=\mathcal{C}=\left\{C^{(n)}\right\}_{n \in \mathbb{N}}$ et $\mathcal{A} \otimes \mathcal{B}=\mathcal{D}=\left\{D^{(n)}\right\}_{n \in \mathbb{N}}$, il vient directement que, pour tout $z=\left(z_{1}, \ldots, z_{d}\right) \in\left(\mathbb{R}_{+}^{*}\right)^{d}$,

$$
\begin{aligned}
\frac{1}{n} \log P^{C^{(n)}}(z) & \leq \frac{1}{n} \log P^{D^{(n)}}(z) \leq \\
& \leq \frac{1}{n} \log P^{C^{(n)}}(z)+\frac{1}{n} \log \operatorname{Min}\left(\mu_{A}^{(n)}, \mu_{B}^{(n)}\right) .
\end{aligned}
$$

D'où le résultat en passant à la limite en $n$. $\square$

\subsection{Procédé d'approximation $\mathcal{L P}$ et polarité}

\section{Hypothèses $\mathcal{H}_{1}, \mathcal{H}_{2}$ et $\mathcal{H}$}

Soit $g$ une fonction de $\mathbf{R}^{d}$ dans $\mathbf{R} \cup\{+\infty\}$ qui vérifie :

- soit les hypothèses $\mathcal{H}_{1}$ :

$$
\left\{\begin{array}{l}
\operatorname{dom}(g) \neq \emptyset \\
g \text { continue sur } \operatorname{dom}(g) \text { (pour la topologie trace) }
\end{array}\right.
$$

- soit les hypothèses $\mathcal{H}_{2}$ :

$$
g \text { convexe, propre et SCI. }
$$

On note $g^{*}$ la fonction polaire de $g$

$$
\begin{gathered}
g^{*}(t)=\operatorname{Sup}_{x \in \mathbb{R}^{d}}(\langle t, x\rangle-g(x)) . \\
-109-
\end{gathered}
$$


Soit $\left\{I^{(n)}\right\}_{n \in \mathbb{N}}$ une suite d'ensembles finis non vides de $\mathbb{Z}^{d}$ telle que les sous-ensembles $K^{(n)}$ de $\mathbb{R}^{d}$ :

$$
K^{(n)}=I^{(n)} / n=\left\{x / n: x \in I^{(n)}\right\},
$$

vérifient les hypothèses $\mathcal{H}$

$$
\left\{\begin{array}{l}
K^{(n)} \subset \operatorname{dom}(g), \quad n \in \mathbb{N}, \\
\lim _{n \rightarrow+\infty} K^{(n)} \supset \operatorname{dom}(g) .
\end{array}\right.
$$

On rappelle que

$$
\operatorname{Lim}_{n \rightarrow+\infty} \operatorname{Inf}^{(n)}=\left\{x=\operatorname{Lim}_{n \rightarrow+\infty} x_{n}: x_{n} \in K^{(n)}, n \in \mathbb{N}\right\} .
$$

LeMme 7. - La suite de fonction $\left\{h_{n}\right\}_{n \in \mathbb{N}}$ définie pour tout $t \in \mathbb{R}^{d}$ par

$$
h_{n}(t)=\underset{x \in K^{(n)}}{\operatorname{Max}}(\langle t, x\rangle-g(x))
$$

converge ponctuellement vers $g^{*}$.

Démonstration. - Commençons d'abord par le cas où $f$ vérifie les hypothèses $\mathcal{H}_{1}$.

Soit $t$ fixé vérifiant $g^{*}(t) \neq+\infty$. Pour tout $\varepsilon>0$ donné, il existe $x_{\varepsilon} \in \operatorname{dom}(g)$ tel que

$$
g^{*}(t)-\frac{\varepsilon}{3} \leq\left\langle t, x_{\varepsilon}\right\rangle-g\left(x_{\varepsilon}\right) \leq g^{*}(t) .
$$

La continuité de $g$ en $x_{\varepsilon} \in \operatorname{dom}(g)$ implique qu'il existe $\eta>0$ tel que pour tout $x \in \operatorname{dom}(g)$ vérifiant $\left\|x-x_{\varepsilon}\right\|_{2} \leq \eta$ on ait

$$
\left|g(x)-g\left(x_{\varepsilon}\right)\right| \leq \frac{\varepsilon}{3} \text {. }
$$

Si on impose de plus la condition sur $\eta$ pour que

$$
\left\|x-x_{\varepsilon}\right\|_{2}\|t\|_{2} \leq \frac{\varepsilon}{3}
$$

pour tout $x \in \operatorname{dom}(g)$ tel que $\left\|x-x_{\varepsilon}\right\|_{2} \leq \eta$, nous avons l'inégalité suivante

$$
\begin{aligned}
\left|\left\langle t, x_{\varepsilon}\right\rangle-g\left(x_{\varepsilon}\right)-\{\langle t, x\rangle-g(x)\}\right| & \leq\|t\|_{2}\left\|x-x_{\varepsilon}\right\|_{2}+\left|g(x)-g\left(x_{\varepsilon}\right)\right| \\
& \leq \frac{2 \varepsilon}{3} . \\
- & 110-
\end{aligned}
$$


Des inégalités (6) et (7), il vient que pour tout $x \in \operatorname{dom}(g)$ vérifiant $\left\|x-x_{\varepsilon}\right\|_{2} \leq \eta$ :

$$
g^{*}(t)-\varepsilon \leq\langle t, x\rangle-g(x) \leq g^{*}(t) .
$$

Utilisant les propriétés des ensembles $K^{(n)}$, nous pouvons choisir un entier $N$ tel que pour tout $n \geq N$ il existe $x_{n}$ appartenant à $K^{(n)}$ et donc à $\operatorname{dom}(g)$ tel que

$$
\left\|x_{n}-x_{\varepsilon}\right\|_{2} \leq \eta
$$

et par conséquent pour tout $n \geq N$ :

$$
g^{*}(t)-\varepsilon \leq h_{n}(t) \leq g^{*}(t)
$$

Ce qui termine la démonstration pour $g^{*}(t) \neq+\infty$.

Traitons maitenant le cas où $g^{*}(t)=+\infty$. Pour tout nombre réel $\alpha>0$, il existe $x_{\alpha} \in \operatorname{dom}(g)$ tel que

$$
\left\langle t, x_{\alpha}\right\rangle-g\left(x_{\alpha}\right) \geq 3 \alpha .
$$

Nous pouvons choisir $\eta>0$ tel que pour tout $x \in \operatorname{dom}(g)$ vérifiant $\left\|x-x_{\alpha}\right\|_{2} \leq \eta$ on ait

$$
\left|g(x)-g\left(x_{\alpha}\right)\right| \leq \alpha \quad \text { et } \quad\|t\|_{2}\left\|x-x_{\alpha}\right\|_{2} \leq \alpha .
$$

Et donc, en écrivant

$$
\langle t, x\rangle-g(x)=\left\langle t, x_{\alpha}\right\rangle-g\left(x_{\alpha}\right)-\left\{\left\langle t,\left(x_{\alpha}-x\right)\right\rangle-\left(g\left(x_{\alpha}\right)-g(x)\right)\right\} .
$$

On en déduit que

$$
\langle t, x\rangle-g(x) \geq 3 \alpha-2 \alpha=\alpha .
$$

Les propriétés des $K^{(n)}$ nous permettent de choisir $N$ tel que pour tout $n \geq N$ il existe $x_{n} \in K^{(n)}$ tel que

$$
\left\|x_{n}-x_{\alpha}\right\|_{2} \leq \eta
$$

Donc pour tout $n \geq N$, on a l'inégalité $h_{n}(t) \geq \alpha$ ce qui termine la démonstration. 
Examinons maintenant le cas où la fonction $f$ vérifie les hypothèses $\mathcal{H}_{2}$. Ces hypothèses impliquent donc que $\operatorname{dom}(f)$ est un convexe non vide. Pour le cas où $g^{*}(t)<+\infty$, une démonstration identique peut être faite à condition de choisir le point noté $x_{\varepsilon}$ appartenant à l'intérieur relatif de $\operatorname{dom}(f)$, puisque $f$ est continue par rapport à cet intérieur relatif. On commence donc par choisir $\tilde{\varepsilon}<\varepsilon / 3$ puis $\tilde{x}_{\varepsilon}$ appartenant à $\operatorname{dom}(f)$ et vérifiant une inégalité identique à $(6)$ avec

$$
g^{*}(t)-\dot{\tilde{\varepsilon}} \leq\left\langle t, \tilde{x}_{\varepsilon}\right\rangle-g\left(\tilde{x}_{\varepsilon}\right) \leq g^{*}(t) .
$$

On sait [8] que $f$ est continue sur tout simplexe inclus dans $\operatorname{dom}(f)$. Considérons donc un segment joignant $\tilde{x}_{\varepsilon}$ à un point de l'intérieur relatif de $\operatorname{dom}(f)$. L'intérieur de ce segment est contenu dans l'intérieur relatif de $\operatorname{dom}(f)$ et la fonction $f$ étant continue sur le segment fermé, il est possible de choisir $x_{\varepsilon}$ appartenant à l'intérieur de segment et vérifiant

$$
g^{*}(t)-\frac{\varepsilon}{3} \leq\left\langle t, x_{\varepsilon}\right\rangle-g\left(x_{\varepsilon}\right) \leq g^{*}(t) .
$$

La démonstration peut donc se poursuivre de la même manière. La modification de démonstration est identique pour le cas où $g^{*}(t)=+\infty$. $\square$

La fonction $g$ vérifiant toujours les hypothèses $\mathcal{H}_{1}$ ou $\mathcal{H}_{2}$ et la suite $\left\{I^{(n)}\right\}_{n \in \mathbb{N}}$ les hypothèses $\mathcal{H}$, définissons maintenant la suite de familles positives presque nulles $\mathcal{A}=\left\{A^{(n)}\right\}_{n \in \mathbb{N}}$ par

$$
a_{i}^{(n)}=\left\{\begin{array}{ll}
e^{-n g(i / n)} & \text { si } i \in I^{(n)} \\
0 & \text { sinon }
\end{array} \quad(n \in \mathbb{N}),\right.
$$

et la suite de polynômes GCP associée

$$
P^{A^{(n)}}\left(z_{1}, \ldots, z_{d}\right)=\sum_{i=\left(i_{1}, \ldots, i_{d}\right) \in I^{(n)}} z_{1}^{i_{1}}, \ldots, z_{d}^{i_{d}} e^{-n g(i / n)}, \quad n \in \mathbb{N} .
$$

LEMME 8. - Si

$$
\frac{\log \operatorname{Card}\left(K^{(n)}\right)}{n} \underset{n \rightarrow \infty}{\longrightarrow} 0
$$

alors la suite $\mathcal{A}$ est $\mathcal{L} \mathcal{P}$-convergente et pour tout $\left(t_{1}, \ldots, t_{d}\right) \in \mathbb{R}^{d}$ :

$$
g^{*}\left(t_{1}, \ldots, t_{d}\right)=\operatorname{Lim}_{n \rightarrow \infty} \frac{1}{n} \log P^{A^{(n)}}\left(e^{t_{1}}, \ldots, e^{t_{d}}\right) .
$$


Démonstration. - Rappelons la définition :

$$
h_{n}(t)=\underset{i \in I^{(n)}}{\operatorname{Max}}\left(\left\langle\frac{i}{n}, t\right\rangle-g\left(\frac{i}{n}\right)\right),
$$

et choisissons un entier $i^{(n, t)}$ appartenant à $I^{(n)}$ tel que

$$
h_{n}(t)=\left\langle\frac{i^{(n, t)}}{n}, t\right\rangle-g\left(\frac{i^{(n, t)}}{n}\right) .
$$

Après avoir posé $f_{n}\left(t_{1}, \ldots, t_{d}\right)=(1 / n) \log P^{A^{(n)}}\left(e^{t_{1}}, \ldots, e^{t_{d}}\right)$, nous pouvons écrire en utilisant le lemme 2 :

$$
h_{n}\left(t_{1}, \ldots, t_{d}\right) \leq f_{n}\left(t_{1}, \ldots, t_{d}\right) \leq h_{n}\left(t_{1}, \ldots, t_{d}\right)+\frac{\log \operatorname{Card}\left(I^{(n)}\right)}{n} .
$$

Il a été montré dans le lemme 7 que $\left\{h_{n}\right\}$ converge ponctuellement vers $g^{*}$. On en déduit que la suite $\left\{f_{n}\right\}$ converge ponctuellement vers $g^{*}$.

Exemple. - Soit $g$ définie par

$$
g(x)= \begin{cases}0 & \text { si }|x| \leq 1 \\ +\infty & \text { si }|x|>1\end{cases}
$$

In est clair que $g^{*}(t)=|t|$ pour toute valeur réelle de $t$. Maintenant, nous pouvons choisir

$$
I^{(n)}=\{-n,-n+1, \ldots,-1,0,+1, \ldots, n-1, n\} .
$$

On peut écrire

$$
f_{n}(t)=\frac{1}{n} \log \sum_{i=-n}^{i=+n}\left(e^{t}\right)^{i},
$$

et un calcul direct montre que $f_{n}(t)$ tend vers $|t|$ pour tout $t \in \mathbb{R}$.

THÉOR亡̀me 9. - Soit $f$ une fonction convexe propre $\mathrm{SCI}$ de $\mathbb{R}^{d}$ dans $\mathbb{R} \cup\{+\infty\}$ et $f^{*}$ la fonction conjuguée de $f$. Soit $\left\{I^{(n)}\right\}_{n \in \mathbb{N}}$ une suite de sous-ensembles non vides de $\mathbb{Z}^{d}$ telle que les sous-ensembles $K^{(n)}=I^{(n)} / n$ vérifient :

$$
\left\{\begin{array}{l}
K^{(n)} \subset \operatorname{dom}\left(f^{*}\right), \quad n \in \mathbb{N}, \\
\operatorname{Lim}_{n \rightarrow+\infty} K^{(n)} \supset \operatorname{dom}\left(f^{*}\right), \\
\frac{\log \operatorname{Card}\left(K^{(n)}\right)}{n} \underset{n \rightarrow+\infty}{\longrightarrow} 0 .
\end{array}\right.
$$


La suite $\mathcal{A}=\left\{A^{(n)}\right\}$, définie par

$$
a_{i}^{(n)}=\left\{\begin{array}{ll}
e^{-n f^{*}(i / n)} & \text { si } i \in I^{(n)} \\
0 & \text { sinon }
\end{array} \quad(n \in \mathbb{N})\right.
$$

est $\mathcal{L} \mathcal{P}$-convergente et pour tout $\left(t_{1}, \ldots, t_{d}\right) \in \mathbb{R}^{d}$ :

$$
f\left(t_{1}, \ldots, t_{d}\right)=\operatorname{Lim}_{n \rightarrow \infty} \frac{1}{n} \log P^{A^{(n)}}\left(e^{t_{1}}, \ldots, e^{t_{d}}\right)
$$

Démonstration. - La fonction $f$ étant une fonction convexe propre SCI, $\operatorname{dom}\left(f^{*}\right)$ est donc un convexe non vide et $f^{*}$ est continue sur l'intérieur relatif de son domaine. De plus, on a $f^{* *}=f$. Le théorème est donc une application directe du résultat du lemme 8 , avec $g=f^{*}$ vérifiant les hypothèses $\mathcal{H}_{2}$.

Remarque et exemple. - La propriété précédente nous permet d'énoncer un résultat analogue à celui du théorème 3 . Dans le cas d'une variable, par rapport au théorème 4 , la méthode est non constructive car le calcul des polynômes fait intervenir $f^{*}$ et pas uniquement les valeurs de $f$. Voici sur un exemple la comparaison de ces deux méthodes pour obtenir une telle approximation.

Considérons la fonction $f$ définie par

$$
f(t)= \begin{cases}0 & \text { si }|t| \leq 1 \\ +\infty & \text { si }|t|>1\end{cases}
$$

- Utilisons le procédé développé au cours de la démonstration du théorème 4 et posons

$$
t_{i}^{n}=-1+\frac{2 i}{n}, \quad i=0,1, \ldots, n .
$$

On définit pour tout $t$ :

$$
\ell_{i}^{n}(t)=\lambda_{i}^{n}(t)=0, \quad i=0,1, \ldots, n-1
$$

puis par exemple :

$$
\ell_{-1}^{n}(t)=\lambda_{-1}^{n}(t)=-n(t+1) \quad \text { et } \quad \ell_{n}^{n}(t)=\lambda_{n}^{n}(t)=n(t-1)
$$


On définit le polynôme $P_{n}$ par

$$
P_{n}\left(e^{t}\right)=\sum_{i=-n}^{i=n} e^{n \lambda_{i}^{n}(t)}=e^{-n^{2}}\left(e^{t}\right)^{-n^{2}}+e^{-n^{2}}\left(e^{t}\right)^{n^{2}}+n,
$$

et on vérifie facilement que pour tout $t \in \mathbb{R}$ :

$$
f(t)=\operatorname{Lim}_{n \rightarrow \infty} \frac{1}{n} \log P_{n}\left(e^{t}\right) .
$$

- Utilisons le théorème 9. On a pour tout réel $x, f^{*}(x)=|x|$ et on choisit

$$
I^{(n)}=\left\{-n^{2},-n^{2}+1, \ldots,-1,0,+1, \ldots, n^{2}-1, n^{2}\right\} .
$$

Il est clair que les ensembles $K^{(n)}=I^{(n)} / n$ vérifient

$$
\left\{\begin{array}{l}
K^{(n)} \subset \operatorname{dom}\left(f^{*}\right), \quad n \in \mathbb{N}, \\
\operatorname{Lim}_{n \rightarrow+\infty} K^{(n)} \supset \operatorname{dom}\left(f^{*}\right), \\
\frac{\log \operatorname{Card}\left(K^{(n)}\right)}{n} \underset{n \rightarrow+\infty}{\longrightarrow} 0 .
\end{array}\right.
$$

Les polynômes définis par (8) sont

$$
P_{n}\left(e^{t}\right)=\left(\sum_{i=-n^{2}}^{0} e^{-n f^{*}(i / n)}\left(e^{t}\right)^{i}\right)+\left(\sum_{i=0}^{n^{2}} e^{-n f^{*}(i / n)}\left(e^{t}\right)^{i}\right)-1
$$

ce que l'on peut écrire

$$
P_{n}\left(e^{t}\right)=\left(\sum_{i=0}^{n^{2}}\left\{\left(e^{-t-1}\right)^{i}+\left(e^{t-1}\right)^{i}\right\}\right)-1
$$

Dans le cas où $|t| \neq 1$, on peut écrire

$$
P_{n}\left(e^{t}\right)=\frac{\left(e^{-t-1}\right)^{\left(n^{2}+1\right)}-1}{e^{-t-1}-1}+\frac{\left(e^{t-1}\right)^{\left(n^{2}+1\right)}-1}{e^{t-1}-1}-1 .
$$

Dans le cas où $|t|>1$, cette dernière expression permet de montrer que :

$$
\frac{1}{n} \log P_{n}\left(e^{t}\right) \underset{n \rightarrow+\infty}{\longrightarrow}+\infty .
$$


Dans le cas où $t \in]-1,1[$, on a $-1-t<0$ et $t-1<0$ ce qui permet d'affirmer que

$$
P_{n}\left(e^{t}\right) \underset{n \rightarrow+\infty}{\longrightarrow} 1,
$$

et donc que

$$
\frac{1}{n} \log P_{n}\left(e^{t}\right) \underset{n \rightarrow+\infty}{\longrightarrow} 0
$$

Les résultats pour les valeurs de $t= \pm 1$ s'obtiennent sans difficulté à partir de l'expression (9) des polynômes $P_{n}$.

1.7. Analogies entre les propriétés des fonctions polaires et les propriétés des suites $\mathcal{L P}$-convergentes de familles positives presque nulles

Dans ce paragraphe, on signale, sans démonstration complète, quelques analogies entre les résultats énoncés dans les propriétés 6 et des propriétés classiques des fonctions convexes $([5],[8])$.

On considère dans tout le paragraphe la même suite $\left\{I^{(n)}\right\}_{n \in \mathbb{N}}$ de sousensembles finis et non vides de $\mathbb{Z}^{d}$ et les ensembles $K^{(n)}=I^{(n)} / n$ associés. $\grave{A}$ une fonction $g$ de $\mathbb{R}$ dans $R \cup\{+\infty\}$, telle que $\operatorname{dom}(g)$ soit non vide et contienne $K^{(n)}$ pour tout $n \in \mathbb{N}$, on associe la suite de familles positives presque nulles $\mathcal{T}(g)=\left\{T(g)^{(n)}\right\}_{n \in \mathbb{N}}$, la famille $T(g)^{(n)}$ possédant comme éléments non nuls les seuls éléments :

$$
T(g)_{i}^{(n)}=e^{-n g(i / n)}, \quad i \in I^{(n)},
$$

Lorsque $\mathcal{T}(g)$ est $\mathcal{L P}$-convergente on pose, avec les notations du paragraphe 1.1,

$$
f^{\mathcal{T}(g)}\left(t_{1}, \ldots, t_{d}\right)=\operatorname{Lim}_{n \rightarrow+\infty} \frac{1}{n} \log P^{T(g)^{(n)}}\left(e^{t_{1}}, \ldots, e^{t_{d}}\right) .
$$

On considère deux fonctions $g_{1}$ et $g_{2}$ vérifiant les conditions imposées plus haut à $g$. On se place dans le cas où les suites $\mathcal{T}\left(g_{1}\right)$ et $\mathcal{T}\left(g_{2}\right)$ sont $\mathcal{L} \mathcal{P}$-convergentes et où les ensembles $K^{(n)}$ et les fonctions $g_{1}$ et $g_{2}$ vérifient de plus les hypothèses du lemme 8 qui se traduisent par

$$
f^{\mathcal{T}\left(g_{1}\right)}=g_{1}^{*} \quad \text { et } \quad f^{\mathcal{T}\left(g_{2}\right)}=g_{2}^{*} .
$$


Opérations $\operatorname{Max}\left\{\mathcal{T}\left(g_{1}\right), \mathcal{T}\left(g_{2}\right)\right\}$ et $\operatorname{Min}\left\{\mathcal{T}\left(g_{1}\right), \mathcal{T}\left(g_{2}\right)\right\}$

De la définition de $\mathcal{T}\left(g_{1}\right)$ et $\mathcal{T}\left(g_{2}\right)$, on déduit

$$
\operatorname{Max}\left\{\mathcal{T}\left(g_{1}\right), \mathcal{T}\left(g_{2}\right)\right\}=\mathcal{T}\left(\operatorname{Min}\left(g_{1}, g_{2}\right)\right)
$$

et la propriété

$$
f^{\operatorname{Max}\left\{\mathcal{T}\left(g_{1}\right), \mathcal{T}\left(g_{2}\right)\right\}}=\operatorname{Max}\left(f^{\mathcal{T}\left(g_{1}\right)}, f^{\mathcal{T}\left(g_{2}\right)}\right),
$$

énoncée dans le paragraphe 1.5 (propriétés 6), se traduit si on peut appliquer le résultat du lemme 8 à $\operatorname{Min}\left(g_{1}, g_{2}\right)$ par

$$
\left(\operatorname{Min}\left(g_{1}, g_{2}\right)\right)^{*}=\operatorname{Max}\left(g_{1}^{*}, g_{2}^{*}\right) \text {. }
$$

De même, on a

$$
\operatorname{Min}\left\{\mathcal{T}\left(g_{1}\right), \mathcal{T}\left(g_{2}\right)\right\}=\mathcal{T}\left(\operatorname{Max}\left(g_{1}, g_{2}\right)\right)
$$

et la propriété

$$
f^{\operatorname{Min}\left\{\mathcal{T}\left(g_{1}\right), \mathcal{T}\left(g_{2}\right)\right\}} \leq \operatorname{Min}\left(f^{\mathcal{T}\left(g_{1}\right)}, f^{\mathcal{T}\left(g_{2}\right)}\right)
$$

se traduit, si on peut appliquer le résultat du lemme 8 à $\operatorname{Max}\left(g_{1}, g_{2}\right)$, par

$$
\left(\operatorname{Max}\left(g_{1}, g_{2}\right)\right)^{*} \leq \operatorname{Min}\left(g_{1}^{*}, g_{2}^{*}\right) .
$$

Ce sont deux résultats classiques.

Opération $\mathcal{T}\left(g_{1}\right) \hat{\nabla} \mathcal{T}\left(g_{2}\right)$

Avec l'hypothèse

$$
\frac{\log \operatorname{Card}\left(K^{n}\right)}{n} \underset{n \rightarrow \infty}{\longrightarrow} 0,
$$

la propriété 6 iii) nous permet d'affirmer que $\mathcal{T}\left(g_{1}\right) \hat{\nabla} \mathcal{T}\left(g_{2}\right)$ est $\mathcal{L P}$-convergente, puis que

$$
f^{\mathcal{T}\left(g_{1}\right) \widehat{\nabla} \mathcal{T}\left(g_{2}\right)}=f^{\mathcal{T}\left(g_{1}\right)}+f^{\mathcal{T}\left(g_{2}\right)} .
$$

Si on pose

$$
e^{-n h(i / n)}=\operatorname{Max}_{j+k=i} e^{-n g_{1}(j / n)} e^{-n g_{2}(k / n)},
$$

et donc

$$
h\left(\frac{i}{n}\right)=\underset{j+k=i}{\operatorname{Min}}\left(g_{1}\left(\frac{j}{n}\right)+g_{2}\left(\frac{k}{n}\right)\right),
$$


on remarque que l'opération $\widehat{\nabla}$ est l'équivalent d'une Inf-Convolution discrète et la propriété $h^{*}=g_{1}^{*}+g_{2}^{*}$ serait à rapprocher des propriétés de l'Inf-Convolution.

I.8. Comportement asymptotique des coefficients des polynômes intervenant dans le procédé d'approximation $\mathcal{L P}$

Sur de nombreux exemples appliqués, en particulier dans le domaine de la Physique Statistique qui est à l'origine de ce travail, les coefficients $a_{i}^{(n)}$ intervenant dans une approximation $\mathcal{L P}$ d'une fonction $f$ semblent posséder des propriétés de régularité [3]. Dans ce paragraphe, nous montrons comment le comportement asymptotique des coefficients $a_{i}^{(n)}$ peut être relié à la fonction polaire $f^{*}$.

Notations et hypothèses. - Considérons une famille $\left\{I^{(n)}\right\}_{n \in \mathbb{N}}$ de sousensembles finis non vides de $\mathbb{Z}^{d}$ à laquelle on associe une suite de familles positives presque nulles $\mathcal{A}=\left\{A^{(n)}\right\}_{n \in \mathbb{N}}$, les seuls termes non nuls de la famille $A^{(n)}$ étant les termes $a_{i}^{(n)}$. d'indice $i$ appartenant à $I^{(n)}$. On suppose que la suite $\mathcal{A}$ est $\mathcal{L P}$-convergente vers $f^{\mathcal{A}}$, c'est-à-dire que pour tout $\left(t_{1}, \ldots, t_{d}\right) \in \mathbb{R}^{d}$ :

$f^{\mathcal{A}}\left(t_{1}, \ldots, t_{d}\right)=\operatorname{Lim}_{n \rightarrow \infty} \frac{1}{n} \log \left(\sum_{\left(i_{1}, \ldots, i_{d}\right) \in I^{(n)}}\left(e^{t_{1}}\right)^{i_{1}} \ldots\left(e^{t_{d}}\right)^{i_{d}} a_{\left(i_{1}, \ldots, i_{d}\right)}^{(n)}\right)$.

ThÉORÈme 10.- Supposons qu'il existe une fonction $g$ de $\mathbb{R}^{d}$ dans $\mathbf{R} \cup\{+\infty\}$ satisfaisant aux hypothèses $\mathcal{H}_{1}$ ou $\mathcal{H}_{2}$ du paragraphe 1.5 et telle que les ensembles $K^{(n)}=I^{(n)} / n$ vérifient :

$$
\left\{\begin{array}{l}
K^{(n)} \subset \operatorname{dom}(g), \quad n \in \mathbb{N}, \\
\operatorname{Lim}_{n \rightarrow+\infty} K^{(n)} \supset \operatorname{dom}(g), \\
\frac{\log \operatorname{Card}\left(K^{(n)}\right)}{n} \underset{n \rightarrow \infty}{\longrightarrow} 0 .
\end{array}\right.
$$

Si

$$
\underset{i \in I^{(n)}}{\operatorname{Max}}\left|\frac{1}{n} \log a_{i}^{(n)}+g\left(\frac{i}{n}\right)\right| \underset{n \rightarrow \infty}{\longrightarrow} 0,
$$

alors $g^{*}=f^{\mathcal{A}}$. 
Démonstration. - Soit $t \in \operatorname{dom}(g)$ et $\varepsilon>0$. Utilisant les hypothèses (10), nous pouvons choisir $N_{t, \varepsilon}$ tel que pour tout $n \geq N_{t, \varepsilon}$ et tout $i \in I^{(n)}$ :

$$
-g\left(\frac{i}{n}\right)-\frac{\varepsilon}{2} \leq \frac{1}{n} \log a_{i}^{(n)} \leq-g\left(\frac{i}{n}\right)+\frac{\varepsilon}{2},
$$

et

$$
\left\langle\frac{i}{n}, t\right\rangle-g\left(\frac{i}{n}\right)-\frac{\varepsilon}{2} \leq \frac{1}{n} \log a_{i}^{(n)}+\left\langle\frac{i}{n}, t\right\rangle \leq\left\langle\frac{i}{n}, t\right\rangle-g\left(\frac{i}{n}\right)+\frac{\varepsilon}{2} .
$$

En notant

$$
h_{n}(t)=\underset{i \in I^{(n)}}{\operatorname{Max}}\left(\left\langle\frac{i}{n}, t\right\rangle t-g\left(\frac{i}{n}\right)\right),
$$

il est facile de montrer alors que

$$
\left|\operatorname{Max}_{i \in I^{(n)}}\left(\frac{1}{n} \log a_{i}^{(n)}+\left\langle\frac{i}{n}, t\right\rangle\right)-h_{n}(t)\right| \leq \frac{\varepsilon}{2} .
$$

De plus, l'application du lemme 2 montre que

$$
\begin{aligned}
& \underset{\left(i_{1}, \ldots, i_{d}\right) \in I^{(n)}}{\operatorname{Max}}\left(\frac{1}{n} \log \left(\left(e^{t_{1}}\right)^{i_{1}} \ldots\left(e^{t_{d}}\right)^{i_{d}} a_{\left(i_{1}, \ldots, i_{d}\right)}^{(n)}\right)\right) \leq \\
& \leq \frac{1}{n} \log \sum_{\left(i_{1}, \ldots, i_{d}\right) \in I^{(n)}}\left(e^{t_{1}}\right)^{i_{1}} \ldots\left(e^{t_{d}}\right)^{i_{d}} a_{\left(i_{1}, \ldots, i_{d}\right)}^{(n)} \leq \\
& \leq \quad \underset{\left(i_{1}, \ldots, i_{d}\right) \in I^{(n)}}{\operatorname{Max}}\left(\frac{1}{n} \log \left(\left(e^{t_{1}}\right)^{i_{1}} \ldots\left(e^{t_{d}}\right)^{i_{d}} a_{\left(i_{1}, \ldots, i_{d}\right)}^{(n)}\right)\right)+ \\
& \quad+\frac{\log \operatorname{Card}\left(K^{(n)}\right)}{n},
\end{aligned}
$$

ce qui mène à l'inégalité suivante valable pour tout $n \geq N_{t, \varepsilon}$ :

$$
\left|h_{n}(t)-\frac{1}{n} \log \sum_{\left(i_{1}, \ldots, i_{d}\right) \in I^{(n)}} e^{t_{1}} \ldots e^{t_{d}} a_{\left(i_{1}, \ldots, i_{d}\right)}^{(n)}\right| \leq \frac{\varepsilon}{2}+\frac{\log \operatorname{Card}\left(K^{(n)}\right)}{n} .
$$

Le lemme 7 nous assure que $h_{n}$ converge ponctuellement vers $g^{*}$, et nous pouvons donc en déduire que $g^{*}=f^{\mathcal{A}}$.

Exemple. - Supposons d'abord que $I^{(n)}=\{-n, n\}$, avec $a_{-n}^{(n)}=a_{n}^{(n)}=1$ les seuls termes non nuls de la suite $a^{(n)}$. On peut poser

$$
g(t)= \begin{cases}0 & \text { si } t= \pm 1 \\ +\infty & \text { sinon }\end{cases}
$$


II est facile de montrer que $f_{n}(t)=(1 / n) \log \left(\left(e^{t}\right)^{n}+\left(e^{-t}\right)^{n}\right)$ tend vers $f(t)=|t|$ lorsque $n$ tend vers l'infini. On vérifie bien que $g^{*}=f$, mais dans ce cas $f^{*}=g^{* *} \neq g$. Si nous choisissons maintenant :

$$
I^{(n)}=\{-n,-n+1, \ldots,-1,0,1, \ldots, n-1, n\},
$$

et $a_{i}^{(n)}=1$ pour tout $i$ appartenant à $I^{(n)}$, nous pouvons choisir :

$$
g(t)= \begin{cases}0 & \text { si } t \in[-1,1] \\ +\infty & \text { sinon }\end{cases}
$$

La fonction $f_{n}(t)=(1 / n) \log \sum_{i \in I^{(n)}}\left(e^{t}\right)^{i}$ possède la même limite $f(t)=|t|$ lorsque $n$ tend vers l'infini. Nous avons bien $g^{*}=f$, et de plus dans ce cas $g=f^{*} \operatorname{car} g^{* *}=g$.

\section{Le procédé $\mathcal{L P}$ et les puissances de matrices polynomiales}

\subsection{Introduction}

Dans le cas d'une variable, un cas important dans la construction d'une suite $\left\{P_{n}\right\}_{n \in \mathbb{N}}$ de polynômes GCP, telle que $(1 / n) \log P_{n}\left(e^{t}\right)$ admette une limite lorsque $n$ tend vers l'infini, est celui où :

$$
P_{n}(z)=\operatorname{Trace}(A(z))^{n}
$$

$A(z)$ étant une matrice de $\mathcal{M}_{n, n}(\mathbb{R}[z])$ à coefficients polynomiaux de la forme

$$
A(z)=\left(z^{m_{i, j}}\right), \quad m_{i, j} \in \mathbb{N}(i, j=1,2, \ldots, n) .
$$

Dans ce cas, $z=e^{t}$ est alors strictement positif et la matrice $A\left(e^{t}\right)$ admet une valeur propre strictement positive $\lambda(t)$ de plus grand module d'après le théorème de Perron-Frobenius. On a alors pour tout $t$ appartenant à $\mathbb{R}$ :

$$
f(t)=\operatorname{Lim}_{n \rightarrow \infty} \frac{1}{n} \log P_{n}\left(e^{t}\right)=\log \lambda(t) .
$$

On peut montrer directement, en utilisant des propriétés de $\lambda(t)$, que la fonction $f$ de $R$ dans $R$ ainsi définie est analytique en tout point. Le théorème 5 associé au corollaire 16 de localisation des zéros des polynômes 
$P_{n}(z)$, établis dans la suite de cette section, nous permettent de donner une autre démonstration de cette propriété. En effet dans cette section, nous allons montrer l'absence de zéros des polynômes Trace $(A(z))^{n}$ au voisinage de l'axe réel positif. En fin de section, nous montrerons un autre résultat de localisation des zéros de ces polynômes pouvant être étendu à des cas généraux de matrices polynomiales (théorème 17). Ces résultats de localisation sont très particuliers parmi les propriétés générales concernant les racines des polynômes [6].

\subsection{Un théorème de localisation de racines en secteur}

\subsubsection{Propriétés de quelques combinaisons de nombres complexes}

Notations. - Dans le plan complexe $\mathbb{C}$ et pour tout $\gamma \in[0, \pi[, \mathcal{S}(\gamma)$ désignera l'ensemble des secteurs $S_{\gamma}$ fermés, privés de l'origine et d'ouverture $\gamma$. Un secteur $S_{\gamma}$ sera donc de la forme

$$
S_{\gamma}=\{z \in \mathbb{C}: \omega \leq \arg z \leq \omega+\gamma \text { et } z \neq 0\},
$$

$\omega$ étant un nombre réel quelconque.

Dans l'exposé qui suit, on indiquera entre crochets, sous la forme $S_{\gamma}[\cdot]$, une liste de paramètre dont dépend éventuellement le secteur $S_{\gamma}$.

Lemme 11. - Soient $\theta$ et $\phi$ deux nombres réels positifs de somme inférieure ou égale $\grave{a} \pi / 2$ et une famille $\mathcal{Z}=\left\{z_{j}\right\}_{j=1,2, \ldots, p}$ de $p$ nombres complexes appartenant $\dot{a}$ un secteur $S_{\theta}$ de $\mathcal{S}(\theta)$.

Il existe un secteur $S_{\phi}[\mathcal{Z}]$ appartenant à $\mathcal{S}(\phi)$ tel que pour toute famille de $p$ nombres réels $\left\{\phi_{j}\right\}_{j=1,2, \ldots, p}$ vérifiant :

$$
0 \leq \phi_{j} \leq \phi, \quad j=1,2, \ldots, p,
$$

$\sum_{j=1}^{p} z_{j} e^{i \phi_{j}}$ appartient à $S_{\phi}[\mathcal{Z}]$. Le secteur $S_{\phi}[\mathcal{Z}]$ s'appuie sur l'isobarycentre des points de $\mathcal{Z}$ et son transformé par rotation d'angle $\phi$.

Démonstration. - On pose

$$
\left|z_{j}\right|=r_{j} \quad \text { et } \quad \arg z_{j}=\theta_{j}, \quad j=1,2, \ldots, p,
$$

et par une rotation, on se ramène au cas où $\phi+\theta_{j} \in[0, \pi / 2]$, $j=1,2, \ldots, p$. 
On remarque pour commencer que tous les nombres $z_{j} e^{i \phi_{j}}$ sont non nuls, appartiennent à un secteur $S_{\pi / 2}$, et que leur somme ne peut donc être nulle. D'autre part, on a

$$
\begin{aligned}
\sum_{j=1}^{p} z_{j} e^{i \phi_{j}} & =\sum_{j=1}^{p} r_{j} e^{i\left(\phi_{j}+\theta_{j}\right)} \\
& =\sum_{j=1}^{p} r_{j} \cos \left(\phi_{j}+\theta_{j}\right)+i \sum_{j=1}^{p} r_{j} \sin \left(\phi_{j}+\theta_{j}\right) .
\end{aligned}
$$

Il est clair alors que dans $[0, \pi / 2]$ où varie $\phi_{j}+\theta_{j}$, les parties réelle et imaginaire de la somme précédente sont respectivement décroissante et croissante en $\phi_{j}+\theta_{j}$. Le nombre complexe de la forme précédente de plus grand argument est alors $\sum_{j=1}^{p} z_{j} e^{i \phi}$, et celui de plus petit argument est $\sum_{j=1}^{p} z_{j}$. On peut remarquer que le secteur ainsi défini s'appuie sur l'isobarycentre des points de $\mathcal{Z}$ et son transformé par rotation d'angle $\phi$.

LEMME 12. - Soient une famille $\mathcal{Z}=\left\{z_{j}\right\}_{j=1,2, \ldots, p}$ de $p$ nombres complexes appartenant $\grave{a}$ un secteur $S_{\theta}$ de $\mathcal{S}(\theta)$ avec $\theta \in[0, \pi / 2[$. On désignera par $m$ et $M$ deux nombres réels vérifiant:

$$
0<\boldsymbol{m} \leq M
$$

Il existe un nombre réel $k \in[0,1$ [ ne dépendant que de $m, M$ et $\theta$, et un secteur $S_{k \theta}[\mathcal{Z}]$ de $\mathcal{S}(k \theta)$ tels que pour toute famille $\left\{m_{j}\right\}_{j=1,2, \ldots, p}$ de $p$ nombres réels vérifiant:

$$
m \leq m_{j} \leq M, \quad j=1,2, \ldots, p
$$

$\sum_{j=1}^{p} m_{j} z_{j}$ appartienne $\grave{a} S_{k \theta}[\mathcal{Z}]$

Démonstration. - $\mathrm{Si} \theta=0$, la démonstration est triviale et on peut choisir $k=0$. Si $\theta \in] 0, \pi / 2[$, on pose

$$
\left|z_{j}\right|=r_{j} \quad \text { et } \quad \arg z_{j}=\theta_{j}, \quad j=1,2, \ldots, p
$$

et par une rotation, on se ramène au cas où les angles $\theta_{j}$ appartiennent à $[0, \theta]$.

Remarquons tout d'abord que la somme $\sum_{j=1}^{p} m_{j} z_{j}$ ne peut être nulle. On étudie ensuite l'argument $\phi\left(m_{1}, m_{2}, \ldots, m_{p}\right)$ du nombre complexe 
$\sum_{j=1}^{p} m_{j} z_{j}$. La tangente de cet argument est une fonction continue de $\left(m_{1}, m_{2}, \ldots, m_{p}\right)$ définie par

$$
\tan \phi\left(m_{1}, m_{2}, \ldots, m_{p}\right)=\frac{\sum_{j=1}^{p} m_{j} r_{j} \sin \theta_{j}}{\sum_{j=1}^{p} m_{j} r_{j} \cos \theta_{j}} .
$$

Supposons que son minimum soit réalisé pour $\left(\nu_{1}, \nu_{2}, \ldots, \nu_{p}\right)$ et son maximum pour $\left(\mu_{1}, \mu_{2}, \ldots, \mu_{p}\right)$, et notons $\phi_{\nu}$ et $\phi_{\mu}$ les angles correspondants. On peut écrire :

$$
\tan \left(\phi_{\mu}-\phi_{\nu}\right)=\frac{\tan \phi_{\mu}-\tan \phi_{\nu}}{1+\tan \phi_{\mu} \tan \phi_{\nu}}
$$

ce qui est égal à

$$
\frac{\mathbf{K}_{\nu_{\mathrm{c}}} \mathbf{K}_{\mu_{\mathrm{s}}}-\mathbf{K}_{\mu_{\mathrm{c}}} \mathbf{K}_{\nu_{\mathrm{s}}}}{\mathbf{K}_{\nu_{\mathrm{c}}} \mathbf{K}_{\mu_{\mathrm{c}}}+\mathbf{K}_{\nu_{\mathrm{s}}} \mathbf{K}_{\mu_{\mathrm{s}}}}
$$

avec

$$
\begin{cases}\mathbf{K}_{\mu_{\mathrm{c}}}=\left(\sum_{1 \leq j \leq p} \mu_{j} r_{j} \cos \theta_{j}\right), & \mathbf{K}_{\mu_{\mathrm{s}}}=\left(\sum_{1 \leq j \leq p} \mu_{j} r_{j} \sin \theta_{j}\right), \\ \mathbf{K}_{\nu_{\mathrm{c}}}=\left(\sum_{1 \leq j \leq p} \nu_{j} r_{j} \cos \theta_{j}\right), & \mathbf{K}_{\nu_{\mathrm{s}}}=\left(\sum_{1 \leq j \leq p} \nu_{j} r_{j} \sin \theta_{j}\right),\end{cases}
$$

ce qui peut encore s'écrire :

$$
\frac{\sum_{1 \leq i<j \leq p} r_{i} r_{j}\left(\nu_{i} \mu_{j}-\nu_{j} \mu_{i}\right) \sin \left(\theta_{j}-\theta_{i}\right)}{\sum_{1 \leq i \leq p} \nu_{i} \mu_{i} r_{i}^{2}+\sum_{1 \leq i<j \leq p} r_{i} r_{j}\left(\nu_{i} \mu_{j}+\nu_{j} \mu_{i}\right) \cos \left(\theta_{j}-\theta_{i}\right)} .
$$

Il est clair que $\tan \left(\phi_{\mu}-\phi_{\nu}\right)$ est positif et donc que

$$
\begin{aligned}
0 & \leq \tan \left(\phi_{\mu}-\phi_{\nu}\right) \leq \\
& \leq \frac{\sum_{1 \leq i<j \leq p} r_{i} r_{j}\left|\nu_{i} \mu_{j}-\nu_{j} \mu_{i}\right| \sin \left|\theta_{j}-\theta_{i}\right|}{\sum_{1 \leq i<j \leq p} r_{i} r_{j}\left(\nu_{i} \mu_{j}+\nu_{j} \mu_{i}\right) \cos \left(\theta_{j}-\theta_{i}\right)} \leq \\
& \leq \operatorname{Max}_{1 \leq i<j \leq p}\left|\frac{\nu_{i} \mu_{j}-\nu_{j} \mu_{i}}{\nu_{i} \mu_{j}+\nu_{j} \mu_{i}}\right| \tan \theta,
\end{aligned}
$$


et finalement

$$
0 \leq \tan \left(\phi_{\mu}-\phi_{\nu}\right) \leq\left(\frac{M^{2}-m^{2}}{M^{2}+m^{2}}\right) \tan \theta
$$

On détermine alors $k \in[0,1[$ tel que

$$
\tan k \theta=\left(\frac{M^{2}-m^{2}}{M^{2}+m^{2}}\right) \tan \theta
$$

ce qui termine la preuve.

THÉORÈmE 13. - Soient $\theta$ et $\phi$ deux nombres réels positifs vérifiant $\theta+\phi \in\left[0, \pi / 2\left[\right.\right.$ et une famille $\mathcal{Z}=\left\{z_{j}\right\}_{j=1,2, \ldots, p}$ de $p$ nombres complexes appartenant à un secteur $S_{\theta}$ de $\mathcal{S}(\theta)$. Étant donnés deux nombres réels $m$ et $M$ vérifiant $0<m \leq M$, il existe un secteur $S_{\phi+k \theta}[\mathcal{Z}]$ de $\mathcal{S}(\phi+k \theta)$, avec $k \in[0,1$ [ ne dépendant que de $m, M$ et $\theta$, tel que pour toute famille $\left\{\alpha_{j}\right\}_{j=1,2, \ldots, p}$ de $p$ nombres complexes vérifiant

$$
0 \leq \arg \alpha_{j} \leq \phi \quad \text { et } \quad m \leq\left|\alpha_{j}\right| \leq M, \quad i=1,2, \ldots, p,
$$

le nombre complexe $\sum_{j=1}^{p} z_{j} \alpha_{j}$ appartienne à $S_{\phi+k \theta}[\mathcal{Z}]$.

Démonstration. - On a $\theta \in[0, \pi / 2$ [. Par l'application du lemme 12, on montre que les points $\sum_{j=1}^{p} z_{j}\left|\alpha_{j}\right|$ appartiennent à un secteur $S_{k \theta}[\mathcal{Z}]$ de $\mathcal{S}(k \theta)$ avec $k \in[0,1$ [. En utilisant le lemme 11, il vient que les points $\sum_{j=1}^{p} z_{j}\left|\alpha_{j}\right| e^{i \text { arg } \alpha_{j}}$ appartiennent au secteur s'appuyant sur le centre de gravité des points $\left\{z_{j}\left|\alpha_{j}\right|\right\}_{j=1,2, \ldots, p}$ et son transformé par la rotation d'angle $\phi$. Il en résulte donc que les points $\sum_{j=1}^{p} z_{j}\left|\alpha_{j}\right| e^{i \arg \alpha_{j}}$ appartiennent à un même secteur $S_{\phi+k \theta}[\mathcal{Z}]$ de $\mathcal{S}(\phi+k \theta)$.

\subsubsection{Puissances de certaines matrices à coefficients polynomiaux}

LEMmE 14. - Soit $A(z)$ une matrice de $\mathcal{M}_{p, p}(\mathbb{R}[z])$ de la forme

$$
A(z)=\left(z^{m_{i, j}}\right), \quad m_{i, j} \in \mathbb{N}, \quad i, j=1,2, \ldots, p
$$

On note $A^{n}(z)$ la puissance $n$-ème de la matrice $A(z)$.

Soient $\theta \in] 0, \pi / 2\left[\right.$ et $S_{\theta}$ un secteur de $\mathcal{S}(\theta)$. Soit $V=\left(\nu_{i}\right)$ un vecteur de $\mathbb{C}^{p}$ tel que $\nu_{i} \in S_{\theta}$ pour tout $i \in\{1,2, \ldots, p\}$. 
Fonctions convexes et logarithmes de polynômes à coefficients positifs

Étant donnés deux nombres réels $\alpha$ et $\beta$ vérifiant :

$$
\mathbf{0}<\alpha \leq \beta
$$

il existe $\gamma>\mathbf{0}$ tel que pour tout $n \in \mathbb{N}^{*}$ et pour tout $z$ vérifiant

$$
\alpha \leq|z| \leq \beta \quad \text { et } \quad 0 \leq \arg z \leq \gamma,
$$

toutes les composantes du vecteur $A^{n}(z) V$ appartiennent $\grave{a}$ un même secteur $S_{\theta}[V, n]$ (dépendant de $V$ et de $n$ mais d'ouverture $\theta$ fixée).

On peut énoncer le même résultat pour le vecteur (ligne) ${ }^{\mathrm{t}} V A^{n}(z)$ $\left({ }^{\mathrm{t}} \mathrm{V}\right.$ désignant le vecteur ligne transposé de $\left.V\right)$.

Démonstration. - Nous avons

$$
A(z) V=\left(\sum_{j=1}^{p} z^{m_{i j}} \nu_{j}\right)_{i=1,2, \ldots, n} .
$$

Les $m_{i, j}$ étant en nombre fini, il existe $m$ et $M$ vérifiant $0<m \leq M$ tels que pour tous les $z$ vérifiant $\alpha \leq|z| \leq \beta$ on ait

$$
0<m \leq\left|z^{m_{i, j}}\right| \leq M, \quad i, j=1,2, \ldots, p
$$

Et de plus pour tout $\phi>0$, il existe $\gamma>0$ tel que :

$$
0 \leq \arg z \leq \gamma \Rightarrow 0 \leq \arg z^{m_{i, j}} \leq \phi, \quad i, j=1,2, \ldots, p .
$$

Utilisant la détermination de $k$ du théorème 13 relative à $m, M$ et $\theta$, choisissons $\phi$, tel que $\phi+k \theta \leq \theta$, ainsi qu'une valeur de $\gamma$ déterminée par l'implication (11.2). Toutes les composantes de $A(z) V$ appartiennent alors à un même secteur $S_{\theta}[V, 1]$. Pour les puissances successives de $A(z)$, la démonstration se poursuit alors par récurrence.

THÉORÈME 15. - Soit $A(z)=\left(z^{m_{i, j}}\right)$ une matrice de $\mathcal{M}_{p, p}(\mathbb{R}[z])$ avec

$$
m_{i, j} \in \mathbb{N}, \quad i, j=1,2, \ldots, p
$$


Pour tout réel $\theta \in] 0, \pi / 2[$ et tout couple de réels $(\alpha, \beta)$ vérifiant $\mathbf{0}<\alpha \leq \beta$, il existe $\gamma>0$ tel que pour tout $n \in \mathbb{N}^{*}$ et pour tout $z \in \mathbb{C}$ vérifiant

$$
\alpha \leq|z| \leq \beta \quad \text { et } \quad 0 \leq \arg z \leq \gamma,
$$

tous les coefficients de $A^{n}(z)$ soient dans un même secteur $S_{\theta}[z, n]$ (dépendant de $z$ et de $n$ mais d'ouverture $\theta$ fixée).

Démonstration. - On pose $\varepsilon=\theta / 3$ et en choisissant comme vecteurs $V$ les vecteurs colonnes (ou les vecteurs lignes de $A(z)$ ), on montre qu'il existe $\gamma>0$ tel que pour tout $z$ d'argument appartenant à $[0, \gamma]$ les éléments d'une même ligne $i$ (ou d'une même colonne) de $A^{n}(z)$ appartiennent à un même secteur $S_{\varepsilon}[i, z, n]$ ne dépendant que de la ligne $i$ (ou de la colonne) de $z$ et de $n$.

Par transitivité, il vient que tout les éléments de $A^{n}(z)$ se situent dans un même secteur $S_{3 \varepsilon}[z, n]$.

Corollaire 16. - Soit $A(z)$ une matrice de $\mathcal{M}_{p, p}(\mathbb{R}[z])$ de la forme

$$
A(z)=\left(z^{m_{i, j}}\right), \quad m_{i, j} \in \mathbb{N}, i, j=1,2, \ldots, p .
$$

Pour tout couple de réels $(\alpha, \beta)$ vérifiant $0<\alpha \leq \beta$, il existe $\gamma>0$ tel que pour tout $n \in \mathbb{N}^{*}$ et tout $z \in \mathbb{C}$ vérifiant

$$
\alpha \leq|z| \leq \beta \quad \text { et } \quad-\gamma \leq \arg z \leq \gamma,
$$

on ait :

$$
\operatorname{Trace} A^{n}(z) \neq 0 \text {. }
$$

Démonstration. - Le théorème 15 nous fournit le résultat pour $0 \leq \arg z \leq \gamma$. Le résultat est également vrai pour $\arg z \in[-\gamma, 0]$ car le polynôme Trace $A^{n}(z)$ est à coefficients réels positifs.

Remarque. - On peut énoncer un théorème équivalent pour des matrices de $\mathcal{M}_{p, p}(\mathbb{R}[z])$ dont les coefficients sont des polynômes non identiquement nuls à coefficients positifs. $D$ 'autres variantes sont également possibles [3].

\subsubsection{Exemple}

Illustrons le résultat du théorème 15 sur la matrice suivante :

$$
\begin{aligned}
A(z) & =\left(\begin{array}{ll}
z & 1 \\
1 & z
\end{array}\right) . \\
& -126-
\end{aligned}
$$


Pour tout $n \in \mathbb{N}^{*}$, on a

$$
A^{n}(z)=\frac{1}{2}\left(\begin{array}{ll}
(z+1)^{n}+(z-1)^{n} & (z+1)^{n}-(z-1)^{n} \\
(z+1)^{n}-(z-1)^{n} & (z+1)^{n}+(z-1)^{n}
\end{array}\right)
$$

que l'on écrit pour $z \neq-1$ :

$$
A^{n}(z)=\frac{(z+1)^{n}}{2}\left(\begin{array}{ll}
1+(z-1 / z+1)^{n} & 1-(z-1 / z+1)^{n} \\
1-(z-1 / z+1)^{n} & 1+(z-1 / z+1)^{n}
\end{array}\right),
$$

et on s'intéresse aux coefficients de la matrice après mise en facteur de $(z+1)^{n} / 2$.

On se donne donc $\theta \in] 0, \pi / 2[$ et $\alpha, \beta$ vérifiant $0<\alpha \leq \beta$.

Soit $\gamma_{0}$ quelconque appartenant à $] 0, \pi / 2[$. En posant $r=|z|$, $\phi=\arg z \in\left[0, \gamma_{0}\right]$, on peut écrire

$$
\left|\frac{z-1}{z+1}\right|=\sqrt{\frac{r^{2}+1-2 r \cos \phi}{r^{2}+1+2 r \cos \phi}} \leq f_{\gamma_{0}}(r)=\sqrt{\frac{r^{2}+1-2 r \cos \gamma_{0}}{r^{2}+1+2 r \cos \gamma_{0}}}
$$

$\cos \phi$ et $\cos \gamma_{0}$ étant positifs. La fonction $f_{\gamma_{0}}$ est strictement décroissante $\operatorname{sur}[0,1$ [ et strictement croissante sur ] $1,+\infty$ [, et elle admet un minimum positif pour $r=1$. On en déduit donc que pour $\mu_{\gamma_{0}, \alpha, \beta} \in[0,1$ [ vérifiant

$$
\mu_{\gamma_{0}, \alpha, \beta}=\operatorname{Max}\left\{\sqrt{\frac{\alpha^{2}+1-2 \alpha \cos \gamma_{0}}{\alpha^{2}+1+2 \alpha \cos \gamma_{0}}}, \sqrt{\frac{\beta^{2}+1-2 \beta \cos \gamma_{0}}{\beta^{2}+1+2 \beta \cos \gamma_{0}}}\right\},
$$

on a pour tout $r \in[\alpha, \beta]$ :

$$
0 \leq f_{\gamma_{0}}(r) \leq \mu_{\gamma_{0}, \alpha, \beta}<1 .
$$

Pour tout $n \in \mathbb{N}^{*}$ et tout $z$ tel que $|z| \in[\alpha, \beta]$, on a donc

$$
\left|\frac{z-1}{z+1}\right|^{n} \leq\left(\mu_{\gamma_{0}, \alpha, \beta}\right)^{n} .
$$

On peut donc trouver $N_{0}$ tel que pour tout $n>N_{0}$ et tout $z$ tel que $|z| \in[\alpha, \beta]$ et $\arg z \in\left[0, \gamma_{0}\right]:$

$$
-\frac{\theta}{2} \leq \arg \left(1+\left(\frac{z-1}{z+1}\right)^{n}\right) \leq \frac{\theta}{2} \quad \text { et } \quad-\frac{\theta}{2} \leq \arg \left(1-\left(\frac{z-1}{z+1}\right)^{n}\right) \leq \frac{\theta}{2},
$$

et tous les termes de la matrice appartiennent alors à un secteur $S_{\theta}[z, n]$ sans autre condition sur $\gamma_{0}$. 
De plus, pour tout $n \in\left\{1,2, \ldots, N_{0}\right\}$ et tout $z$ réel appartenant à $[\alpha, \beta], 1+(z-1 / z+1)^{n}$ et $1-(z-1 / z+1)^{n}$ sont réels et inclus dans le segment $\left[1-\left(\mu_{0, \alpha, \beta}\right)^{N_{0}}, 1+\left(\mu_{0, \alpha, \beta}\right)^{N_{0}}\right]$ qui ne contient pas l'origine. Pour l'ensemble des valeurs de $n \in\left\{1,2, \ldots, N_{0}\right\}$ en utilisant des propriétés de continuité uniforme en $\phi$ et $r$, on peut donc choisir $\gamma_{n}>0$ tel que pour tout $z$ vérifiant $|z| \in[\alpha, \beta]$ et pour tout $\phi=\arg z$ appartenant à $\left[0, \gamma_{n}\right]$ :

$$
-\frac{\theta}{2} \leq \arg \left(1+\left(\frac{z-1}{z+1}\right)^{n}\right) \leq \frac{\theta}{2} \quad \text { et } \quad-\frac{\theta}{2} \leq \arg \left(1-\left(\frac{z-1}{z+1}\right)^{n}\right) \leq \frac{\theta}{2},
$$

et tous les termes de la matrice $A^{(n)}(z)$ appartiennent alors à un secteur $S_{\theta}[z, n]$. I suffit alors de choisir $\gamma=\operatorname{Min}\left\{\gamma_{0}, \gamma_{1}, \ldots, \gamma_{N_{0}}\right\}$ pour illustrer le théorème 15 .

Remarquons que les racines de Trace $A^{(n)}(z)=0$ sont données par

$$
z_{k}=i \operatorname{cotg}\left(\frac{\pi}{2 n}+\frac{k \pi}{n}\right), \quad k=0,1,2, \ldots, n-1,
$$

et sont donc toutes situées sur l'axe imaginaire. On retrouve facilement avec le calcul fait précédemment, qu'il n'y a acune racine dans le demi-plan $\Re z>0$. En effet, soit $z$ appartenant à ce demi-plan. En posant $r=|z|$, $\phi=\arg z$, il est facile de voir avec les notations précédentes que

$$
\left|\frac{z-1}{z+1}\right|^{n} \leq\left(f_{\phi}(r)\right)^{n} \leq\left(\mu_{\phi, r, r}\right)^{n} \leq \mu_{\phi, r, r}<1,
$$

et $1+(z-1 / z+1)^{n}$ ne peut être nul.

2.3. Un second théorème de localisation des racines des polynômes $P_{n}(z)=\operatorname{Trace}(A(z))^{n}$

Ce théorème s'applique à des matrices à coefficients polynomiaux complexes quelconques. Ce résultat, exprimé de façon diverses, est utilisé en Physique Statistique [1]. On donne ici une version adaptée au cas des puissances de matrices polynomiales.

2.3.1. Notations. - Soit $A(z)$ une matrice de $\mathcal{M}_{p, p}(\mathbb{C}[z])$. On note

$$
\lambda_{1}(z), \lambda_{2}(z), \ldots, \lambda_{p}(z)
$$

les $p$ valeurs propres distinctes ou non de $A(z)$. On note $\mathcal{C}$ l'ensemble des valeurs de $z$ telles que la matrice $A(z)$ admette deux valeurs propres de 
modules égaux et plus grands (ou égaux) que les modules des autres valeurs propres. L'ensemble $\mathcal{C}$ peut donc être écrit

$$
\begin{aligned}
\mathcal{C}=\{z \in \mathbb{C}: \exists i, j \in\{1,2, \ldots, p\} \text { et } i \neq j \mid \\
\left.\left|\lambda_{i}(z)\right|=\left|\lambda_{j}(z)\right| \geq\left|\lambda_{k}(z)\right|, k \in\{1,2, \ldots, p\}\right\} .
\end{aligned}
$$

\subsubsection{Un second théorème de localisation}

THÉORÈme 17. - Soit $\left\{P_{n}\right\}_{n \in \mathbb{N}}$, la famille des polynôme définis par

$$
P_{n}(z)=\operatorname{Trace}(A(z))^{n}, \quad n \in \mathbb{N},
$$

et $\mathcal{R}_{n}$ l'ensemble des racines de $P_{n}(z)$. On a

$$
\underset{n \rightarrow+\infty}{\operatorname{LimSup}} \mathcal{R}_{k}=\bigcap_{n \in \mathbb{N}} \overline{\bigcup_{k \geq n} \mathcal{R}_{k}} \subset \mathcal{C} .
$$

Démonstration. - Désignons par $\lambda_{1}(z)$ l'unique valeur propre de plus grand module lorsqu'elle existe. On pose alors, pour $\alpha \in] 0,1[$ :

$$
\Gamma_{\alpha}=\left\{z \in \mathbb{C}: \alpha\left|\lambda_{1}(z)\right|>\left|\lambda_{k}(z)\right|, k \in\{2,3, \ldots, p\}\right\} .
$$

Notons que $\Gamma_{\alpha}$ est ouvert, en raison de la continuité des coefficients de $A(z)$.

Soit $z \in \Gamma_{\alpha}$. On ne peut avoir $\lambda_{1}(z)=0$ et par conséquent, on peut écrire

$$
P_{n}(z)=\left(\lambda_{1}(z)\right)^{n}\left\{1+\sum_{k=2}^{p}\left(\frac{\lambda_{k}(z)}{\lambda_{1}(z)}\right)^{n}\right\}
$$

ce qui entraîne

$$
\left|P_{n}(z)\right| \geq\left|\lambda_{1}(z)\right|^{n}\left\{1-(p-1) \alpha^{n}\right\} .
$$

Il existe $n(\alpha) \in \mathbb{N}$ tel que pour tout $n \geq n(\alpha)$ :

$$
1-(p-1) \alpha^{p}>0,
$$

et pour tout $n \geq n(\alpha)$, on a donc

$$
\mathcal{R}_{n} \cap \Gamma_{\alpha}=\emptyset .
$$


Par conséquent, pour tout $n \geq n(\alpha)$, on a

$$
\mathcal{R}_{n} \subset \mathrm{C \Gamma}_{\alpha}
$$

et $\Gamma_{\alpha}$ étant ouvert

$$
\bigcup_{k \geq n(\alpha)} \mathcal{R}_{k} \subset C \Gamma_{\alpha} .
$$

Ceci entraîne donc que pour tout $\alpha \in] 0,1[$ :

$$
\bigcap_{n \in \mathbb{N}} \overline{\bigcup_{k \geq n} \mathcal{R}_{k}} \subset \mathcal{C} \Gamma_{\alpha} .
$$

Montrons maintenant que

$$
\bigcap_{\alpha \in] 0,1[} C \Gamma_{\alpha} \subset \mathcal{C}
$$

ou plutôt l'inclusion sur les complémentaires

$$
\operatorname{ccc}\left(\bigcup_{\alpha \in] 0,1[} \Gamma_{\alpha}\right) \text {. }
$$

Si $z \in \complement \mathcal{C}$ cela signifie qu'il existe une valeur propre $\lambda_{1}(z)$ telle que pour tout $k \in\{2,3, \ldots, p\}$ :

$$
\left|\lambda_{1}(z)\right|>\left|\lambda_{k}(z)\right|
$$

Il est alors possible de trouver $\alpha \in] 0,1$ [ tel que $z$ appartienne à $\Gamma_{\alpha}$ et donc à $\bigcup_{\alpha \in] 0,1[} \Gamma_{\alpha}$. On a donc le résultat final :

$$
\bigcap_{n \in \mathbb{N}} \overline{\bigcup_{k \geq n} \mathcal{R}_{k}} \subset \mathcal{C} . \square
$$

\subsubsection{Exemple}

Rappelons la propriété élémentaire suivante :

Soit $p$ et $q \in \mathbb{C}$. Une condition nécessaire et suffisante pour que l'équation du second degré $z^{2}-p z+q=0$, admette deux racines de même module $z^{1}$ et $z^{2}=z^{1} e^{i \phi}(\phi \in \mathbb{R})$ est que $p^{2}=4 q \cos ^{2}(\phi / 2)$. C'est-à-dire qu'il existe $a \in[0,4]$ tel que $p^{2}=a q$. 
L'exemple très simple qui suit, illustre à partir du théorème de localisation 17 , l'absence de racines des polynômes $P_{n}(z)=\operatorname{Trace}(A(z))^{n}$ au voisinage de l'axe réel positif (corollaire 16). Soit la matrice

$$
A(z)=\left(\begin{array}{ccc}
z & z & 1 \\
z & z & 1 \\
1 & 1 & 1
\end{array}\right)
$$

dont les valeurs propres sont $\lambda=0$ et les solutions de l'équation du second degré :

$$
\lambda^{2}-\lambda(2 z+1)+2 z-2=0 .
$$

Pour que ces deux valeurs propres soient de même module il faut et il suffit qu'il existe $a \in[0,4]$ tel que

$$
(2 z+1)^{2}=a(2 z-2) .
$$

Les valeurs de $z$ sont donc solutions de

$$
4 z^{2}-2 z(a-2)+1+2 a=0,
$$

et sont donc

$$
\left(z_{a}^{1}, z_{a}^{2}\right)=\frac{a-2 \pm i \sqrt{a(12-a)}}{4}
$$

pour les valeurs de $a \in[0,4]$. On vérifie aisément que $z_{a}^{1}$ et $z_{a}^{2}$ appartiennent au cercle de centre 1 et de rayon $3 / 2$ et que pour tout $a \in[0,4]$ :

$$
-\frac{1}{2} \leq \Re z_{a}^{1}=\Re z_{a}^{2} \leq \frac{1}{2}
$$

\section{Conclusion}

Dans le présent article, nous avons montré comment construire à partir de suites de logarithmes de polynômes à coefficients positifs, des suites convergentes vers des fonctions convexes $\mathbf{R}^{d}$ dans $R \cup\{+\infty\}$. On a montré que toute fonction convexe SCI était limite de telles suites. Puis, nous avons établi une propriété de caractérisation dans le cas d'une variable. 
Nous avons opté pour le choix de la convergence simple même si certains résultats peuvent s'exprimer en termes d'épi-convergence. De nombreuses questions restent d'ailleurs ouvertes à ce sujet, en particulier celle de déterminer, dans le cas de plusieurs variables, la classe des fonctions convexes pouvant être obtenues comme limite ponctuelle avec le procédé $\mathcal{L P}$. La méthode très constructive, élaborée pour une variable, est peutêtre techniquement généralisable, avec quelques restrictions, à plusieurs variables. Cette étude concerne essentiellement la frontière du domaine effectif de la limite et le comportement de la fonction sur cette frontière. La même question se pose pour d'autre type de convergence. De plus, nous avons constamment utilisé dans cet article, comme intermédiaire entre les logarithmes de polynômes et les fonctions limites, des fonctions égales au maximum d'un nombre fini de fonctionnelles affines. On peut se poser la question de savoir si cet intermédiaire est nécessaire, et il serait assez satisfaisant de pouvoir travailler directement sur les coefficients des polynômes, comme le suggèrent les relations simples montrées entre les polaires de ces fonctions et les coefficients des polynômes. L'utilisation du formalisme des familles presque nulles, même si il ne donne pas de résultats spécifiques dans cet article, est là pour affirmer que l'intérêt d'une telle étude réside, à notre avis, plus en la découverte de relations entre les coefficients de ces familles presque nulles et les fonctions obtenues par le procédé $\mathcal{L P}$, que dans la redécouverte à partir de ces exemples des résultats classiques de l'analyse convexe.

En ce qui concerne les théorèmes de localisation de racines, les théorèmes de localisation des polynômes définis comme les traces de puissances de matrices polynomiales, sont certainement susceptibles de variantes et de généralisations. Le cas de plusieurs variables doit pouvoir donner lieu à certains résultats. D'ailleurs, les rapports entre les singularités des fonctions et les zéros des polynômes, les généralisations, seraient intéressants mais beaucoup plus délicats. Un point intéressant et non étudié ici est également de caractériser les suites de familles positives presque nulles générées par les puissances de matrices polynomiales. On rejoint alors les mêmes perspectives que celles énoncées un peu avant dans cette conclusion. 
Fonctions convexes et logarithmes de polynômes à coefficients positifs

\section{Bibliographie}

[1] Domb (C.) et Green (M.S.) .- Phase transitions and critical phenomena, Vol. 1, Academic Press, 1972.

[2] ISRAËL (R.B.) .- Convexity in the theory of lattice gases, Princeton Series in Physics, 1979.

[3] Lacolle (B.) .- Sur certaines méthodes de calcul de la Physique Statistique, Thèse de Mathématiques, Université de Grenoble 1984.

[4] LACOLlE (B.) .- Approximation d'une fonction convexe lipschitzienne et de ses singularités, M2AN, Vol. 19, 2 (1985).

[5] Laurent (P.-J.) .- Approximation Optimisation, Hermann, 1972.

[6] MARDen (M.) . - The geometry of the zeros of a polynomial in a complex variable, Amer. Math. Soc., Mathematical Survey Number III (1949).

[7] Robert (R.) .- Convergence de fonctionnelles convexes, J. Math. Anal. Appl. 45 (1974), pp. 533-555.

[8] Rockafellar (R.T.) .- Convex Analysis, Princetown University Press, New Jersey, 1970.

[9] Ruelle (D.) .- Statistical Mechanics, The Mathematic Physics Monograph Series, 1969.

[10] Salinetti (G.) et Wets (R. J.-B.) .- On the relation between Two Types of Convergence for Convex Functions, J. Math. Anal. Appl. 60 (1977), pp. 211-226. 